\title{
Killing Two Birds With One Stone - Strain Engineering Facilitates the Development of a Unique Rhamnolipid Production Process
}

\author{
Isabel Bator ${ }^{1,2}$, Tobias Karmainski ${ }^{1,2}$, Till Tiso ${ }^{1,2 *}$ and Lars M. Blank ${ }^{1,2 *}$ \\ ${ }^{1}$ iAMB - Institute of Applied Microbiology, ABBt - Aachen Biology and Biotechnology, RWTH Aachen University, Aachen, \\ Germany, ${ }^{2}$ Bioeconomy Science Center (BioSC), Forschungszentrum Jülich, Jülich, Germany
}

High-titer biosurfactant production in aerated fermenters using hydrophilic substrates is often hampered by excessive foaming. Ethanol has been shown to efficiently destabilize foam of rhamnolipids, a popular group of biosurfactants. To exploit this

OPEN ACCESS

Edited by:

Gloria Soberón-Chávez,

National Autonomous University

of Mexico, Mexico

Reviewed by:

Sujit Jagtap,

University of Illinois

at Urbana-Champaign, United States

Farshad Darvishi,

University of Maragheh, Iran

*Correspondence:

Till Tiso

till.tiso@rwth-aachen.de

Lars M. Blank

lars.blank@rwth-aachen.de

Specialty section:

This article was submitted to

Industrial Biotechnology,

a section of the journal

Frontiers in Bioengineering and

Biotechnology

Received: 25 May 2020

Accepted: 13 July 2020

Published: 07 August 2020

Citation:

Bator I, Karmainski T, Tiso T and Blank LM (2020) Killing Two Birds

With One Stone - Strain Engineering

Facilitates the Development of a

Unique Rhamnolipid Production

Process.

Front. Bioeng. Biotechnol. 8:899

doi: 10.3389/fbioe.2020.00899 feature, we used ethanol as carbon source and defoamer, without introducing novel challenges for rhamnolipid purification. In detail, we engineered the non-pathogenic Pseudomonas putida KT2440 for heterologous rhamnolipid production from ethanol. To obtain a strain with high growth rate on ethanol as sole carbon source at elevated ethanol concentrations, adaptive laboratory evolution (ALE) was performed. Genome re-sequencing allowed to allocate the phenotypic changes to emerged mutations. Several genes were affected and differentially expressed including alcohol and aldehyde dehydrogenases, potentially contributing to the increased growth rate on ethanol of $0.51 \mathrm{~h}^{-1}$ after ALE. Further, mutations in genes were found, which possibly led to increased ethanol tolerance. The engineered rhamnolipid producer was used in a fed-batch fermentation with automated ethanol addition over $23 \mathrm{~h}$, which resulted in a 3-(3-hydroxyalkanoyloxy)alkanoates and mono-rhamnolipids concentration of about $5 \mathrm{~g} \mathrm{~L}^{-1}$. The ethanol concomitantly served as carbon source and defoamer with the advantage of increased rhamnolipid and biomass production. In summary, we present a unique combination of strain and process engineering that facilitated the development of a stable fed-batch fermentation for rhamnolipid production, circumventing mechanical or chemical foam disruption.

Keywords: Pseudomonas, metabolic engineering, synthetic biology, adaptive laboratory evolution, ethanol, rhamnolipid, fermentation, biosurfactants

\section{INTRODUCTION}

Biosurfactants are surface-active molecules with high industrial potential due to their wide range of applications. They can be used in, e.g., bioremediation, enhanced oil recovery, plant protection, cosmetics, and medicine (Lang and Wullbrandt, 1999; Maier and Soberón-Chávez, 2000; Banat et al., 2010; Silva et al., 2014). In contrast to fossil-based surfactants, biosurfactants can be produced from renewable resources, exhibiting often a low ecotoxicological potential (Henkel et al., 2012; Johann et al., 2016). While the number of biosurfactants described is still increasing, they usually 
belong to the classes of glycolipids, phospholipids, lipopeptides, or polymeric biosurfactants (Desai and Banat, 1997).

The glycolipid rhamnolipid was first reported in 1949 by Jarvis and Johnson (Jarvis and Johnson, 1949). The production of rhamnolipids is mainly a trait of the bacterial genera Pseudomonas and Burkholderia (Germer et al., 2020), where the opportunistic pathogen Pseudomonas aeruginosa is one of the most reported species. Rhamnolipids consist of a hydrophobic moiety, one or two $\beta$-hydroxy fatty acids, and a hydrophilic moiety, one or two rhamnose molecules, which are linked through a $\beta$-glycosidic bond to the fatty acid chains (Edwards and Hayashi, 1965; Syldatk et al., 1985). More than 20 different congeners are produced by $P$. aeruginosa, while the most abundant congener contains a $\mathrm{C}_{10}-\mathrm{C}_{10} \beta$-hydroxy fatty acid (Déziel et al., 1999, 2000). For the synthesis, two pathways for the precursor molecules are needed, which exist in Pseudomonas species. The synthesis of dTDP-L-rhamnose is catalyzed by five enzymes, a phosphoglucomutase and four enzymes of the rml-operon, starting from D-glucose-6-phosphate (Rahim et al., 2000). The $\beta$-hydroxyacyl-ACP is synthesized in the fatty acid de novo synthesis. The genes rhlA and rhlB, which are organized in an operon, are involved in monorhamnolipid synthesis (Ochsner et al., 1994; Déziel et al., 2003). The acyltransferase RhlA links two $\beta$-hydroxyacyl-ACP molecules to a dimer called 3-(3-hydroxyalkanoyloxy)alkanoate (HAA). Then, the rhamnosyltransferase RhlB adds a rhamnose to the HAA using dTDP-L-rhamnose as substrate. If one more rhamnose is added by the rhamnosyltransferase RhlC a di-rhamnolipid is produced (Rahim et al., 2001). RhlC is encoded by $r h l C$, which is located in another genomic region of P. aeruginosa.

The production of rhamnolipids with $P$. aeruginosa, although in industry established, encounters some challenges. First, the sophisticated regulation of the rhamnolipid synthesis genes by quorum sensing (Soberón-Chávez et al., 2005; Dusane et al., 2010), which complicates fermentation procedures. Second, the highest rhamnolipid production is achieved using plant oils as substrates (Sim et al., 1997), which increases the effort and costs in downstream processing. Third, the before mentioned pathogenicity is critical for the permission of production processes and the resulting products. Thus, rhamnolipid production with a non-pathogenic host where the production is independent from host intrinsic regulation using renewable hydrophilic substrates is much aimed for. Many studies report the heterologous production of rhamnolipids in different organisms, e.g., Escherichia coli, P. fluorescens, and P. putida KT2440 (Ochsner et al., 1995; Wang et al., 2007; Wittgens et al., 2011). The latter species is a wellestablished chassis in industrial biotechnology and is known for its versatile metabolism and high redox cofactor regeneration rate (Ramos et al., 1995; Nelson et al., 2002; Blank et al., 2010; Tiso et al., 2014).

Apart from the general microbial fermentation challenges, biosurfactant fermentations potentially have extensive foaming and high purification costs as further challenges. Excessive foaming is especially a challenge in aerated and agitated biosurfactant cultivations using hydrophilic substrates. The foaming facilitates biomass and product loss from the liquid phase. The product can be enriched in the foam phase by more than 20 times (Beuker et al., 2014, 2016b). Losing the whole-cell catalyst has a negative impact on volumetric production rate (Davis et al., 2001). While foaming is a true challenge, several strategies to control or prevent foaming exist. Antifoaming agents (or antifoamers) can be used, with the potential drawbacks of lowering the oxygen transfer rate, increasing the cost as they are expensive, and complicate downstream processing due to emulsification (Vardar-Sukan, 1998; Beuker et al., 2014). Another alternative is the use of mechanical foam breakers, which destroy the foam by shear stress (Junker, 2007). These elements have an additional energy demand and need maintenance, thus increasing also process costs. Further alternatives exist, e.g., adsorption of cell-containing foam, bubble-free membrane reactors, and foam fractionation (Winterburn et al., 2011; Beuker et al., 2014; Anic et al., 2017). However, all control or prevention methods have their limits, and usually add complexity to the fermentation. Hence, the development of alternative strategies to simplify the process, e.g., by controlling foaming, is a crucial step in process design and should be addressed in an early stage. Avoiding, e.g., antifoamers reduces elaborative downstream processing and hence costs.

In times of massive production of greenhouse gases, the usage of alternative feedstocks is highly recommended. The overall goal is to establish a circular bioeconomy in which also lignocellulosic biomass and side- and waste streams are used as energy and carbon source. Several studies deal with the production of rhamnolipids from alternative resources, e.g., butane, agroindustrial waste, glycerol, organic acids derived from bio-oil, and xylose (Gehring et al., 2016; Gudiña et al., 2016; Tiso et al., 2017; Arnold et al., 2019; Bator et al., 2020). In the 1990s, ethanol was already considered as an alternative carbon source for rhamnolipid production (Matsufuji et al., 1997). Bioethanol is an interesting substrate because it can be produced from biomass constituents like sugars or sugar polymers (e.g., starch) (Wendisch et al., 2016). But also attempts to use lignocellulosic sugars for bioethanol were made in the last decades, and various cellulosic ethanol plants are in operation (Balan et al., 2013; Duwe et al., 2019; Rosales-Calderon and Arantes, 2019). Further, synthesis gas (syngas) fermentations can be used to produce ethanol from greenhouse gases (Bengelsdorf and Dürre, 2017). A syngas-based industrial-scale ethanol production facility using Clostridium autoethanogenum as biocatalyst was brought into operation in 2018 (LanzaTech, 2018). Hence, $\mathrm{CO}$ and $\mathrm{CO}_{2}$ can directly be used as carbon sources for sustainable ethanol production. The produced ethanol can be used as biofuel, as platform chemical (e.g., ethylene or ethylene glycol synthesis) or as a carbon source for the production of valuable products as proposed here for the synthesis of rhamnolipids.

In this work, we report heterologous rhamnolipid production with engineered $P$. putida KT2440 from ethanol. We used adaptive laboratory evolution (ALE) to improve growth on and tolerance toward ethanol and identified the basis for adaptation by genome re-sequencing. An evolved mutant was characterized 
regarding growth and then engineered for mono-rhamnolipid production. Due to the improved growth parameters of the strain in the presence of ethanol, we were able to develop a unique rhamnolipid production process where ethanol takes over two functions - being the carbon source and the defoaming agent.

\section{MATERIALS AND METHODS}

\section{Strains and Cultivation Conditions}

All bacterial strains used in this study are listed in Table $\mathbf{1}$. E. coli strains, P. putida KT2440 (DSM6125, ATCC47054), and engineered $P$. putida strains were routinely cultivated in lysogeny broth (LB) medium $\left(10 \mathrm{~g} \mathrm{~L}^{-1}\right.$ peptone, $5 \mathrm{~g} \mathrm{~L}^{-1}$ yeast extract, and $10 \mathrm{~g} \mathrm{~L}^{-1} \mathrm{NaCl}$ ). If solid medium was needed, $2 \%(\mathrm{w} / \mathrm{v})$ agar was added. Cultivation of $P$. putida strains was performed at $30^{\circ} \mathrm{C}$ and $E$. coli strains were cultivated at $37^{\circ} \mathrm{C}$. To avoid the loss of plasmids, $50 \mu \mathrm{g} \mathrm{mL}-1$ kanamycin or $30 \mu \mathrm{g} \mathrm{mL} \mathrm{mL}^{-1}$ gentamycin were added to the medium. Quantitative microbiology experiments were performed using M9 minimal medium with a final composition (per L) of $8.5 \mathrm{~g} \mathrm{Na}_{2} \mathrm{HPO}_{4} \cdot 2 \mathrm{H}_{2} \mathrm{O}, 3 \mathrm{~g} \mathrm{KH}_{2} \mathrm{PO}_{4}, 0.5 \mathrm{~g} \mathrm{NaCl}, 1 \mathrm{~g} \mathrm{NH} \mathrm{Nl}_{4}$, $2 \mathrm{mM} \mathrm{MgSO} 4,4.87 \mathrm{mg} \mathrm{FeSO} \cdot 7 \mathrm{H}_{2} \mathrm{O}, 4.12 \mathrm{mg} \mathrm{CaCl} \cdot 2 \mathrm{H}_{2} \mathrm{O}$, $1.5 \mathrm{mg} \mathrm{MnCl}_{2} \cdot 4 \mathrm{H}_{2} \mathrm{O}, 1.87 \mathrm{mg} \mathrm{ZnSO}_{4} \cdot 7 \mathrm{H}_{2} \mathrm{O}, 0.3 \mathrm{mg} \mathrm{H}_{3} \mathrm{BO}_{3}$, $0.25 \mathrm{mg} \mathrm{Na} \mathrm{MoO}_{4} \cdot 2 \mathrm{H}_{2} \mathrm{O}, 0.15 \mathrm{mg} \mathrm{CuCl} \cdot 2 \mathrm{H}_{2} \mathrm{O}, 0.84 \mathrm{mg}$ $\mathrm{Na}_{2}$ EDTA $2 \mathrm{H}_{2} \mathrm{O}$ (Sambrook and Russell, 2001), and $10 \mathrm{~g}$ glucose for pre-cultures or different concentrations of glucose and ethanol for main cultures as indicated. Liquid cultivations were performed in $500 \mathrm{~mL}$ shake flasks with $10 \%$ filling volume at $300 \mathrm{rpm}$ and in 24-deep well plates (SystemDuetz; Enzyscreen B.V., Heemstede, Netherlands) with $1 \mathrm{~mL}$ filling volume at $300 \mathrm{rpm}$ and a throw of $50 \mathrm{~mm}$. A Growth Profiler 960 (Enzyscreen B.V., Heemstede, Netherlands) was used for online analysis of growth without sampling. 96-well plates with $200 \mu \mathrm{L}$ filling volume at $225 \mathrm{rpm}$ were incubated and the density was determined by image analysis.

\section{Adaptive Laboratory Evolution}

For adaptation to ethanol, two consecutive ALE experiments were carried out. Each experiment was performed in two biological replicates: $P$. putida KT2440 was grown in M9 minimal medium containing $2 \mathrm{~g} \mathrm{~L}^{-1}$ glucose and $4 \%(\mathrm{v} / \mathrm{v})$ ethanol. The optical density $\left(\mathrm{OD}_{600}\right)$ was measured and fresh medium was inoculated with a starting $\mathrm{OD}_{600}$ of 0.1 daily. After 30 days (23 transfers), the medium composition was changed to $4 \%$ $(\mathrm{v} / \mathrm{v})$ ethanol. ALE was carried on for twelve more days (10 transfers). To obtain single isolates, the inhomogeneous culture was plated on LB-agar. Subsequently, single colonies were tested for adaptation to ethanol in a 96-well plate in a Growth Profiler 960 (Enzyscreen B.V., Heemstede, Netherlands).

\section{DNA Techniques}

Genome re-sequencing of the wild type, P. putida KT2440 E1, and $P$. putida KT2440 E1.1 was performed to identify mutations obtained by ALE on ethanol. Therefore, genomic DNA was isolated with the High Pure PCR Template Preparation Kit (Roche Holding, Basel, Switzerland). The sequencing was done by Eurofins Genomics (Ebersberg, Germany) using Illumina technology as paired-end reads of 150 base pairs. Single nucleotide polymorphisms (SNPs) and insertions or deletions (InDels), which were analyzed by GATK's Haplotype Caller (McKenna et al., 2010; Depristo et al., 2011), were visualized with the Integrative Genomics Viewer (Robinson et al., 2011). The sequences of the wild type, $P$. putida KT2440 E1, and $P$. putida KT2440 E1.1 were deposited in the Sequence Read Archive with the accession number (PRJNA642834).

Expression levels of genes were determined by quantitative real-time PCR (qRT-PCR). RNA was extracted from the wild type, P. putida KT2440 E1, and P. putida KT2440 E1.1 when growing in M9 minimal medium containing $0.96 \%$ (v/v) ethanol. The medium was inoculated at a starting $\mathrm{OD}_{600}$ of 0.1 and the cells were cultivated until they reached an $\mathrm{OD}_{600}$ of $1.1 \mathrm{~mL}$

TABLE 1 | Bacterial strains used in this study.

\begin{tabular}{|c|c|c|}
\hline Strains and plasmids & Characteristics & References \\
\hline \multicolumn{3}{|l|}{ E. coli } \\
\hline PIR2 & 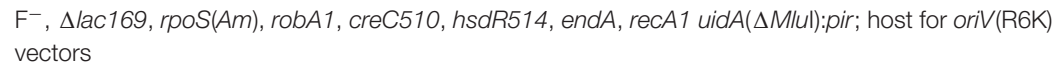 & Thermo Fisher Scientific \\
\hline HB101 pRK2013 & $\mathrm{Sm}^{R}$, hsdR-M+, proA2, leuB6, thi-1, recA; harboring plasmid pRK2013 & Ditta et al., 1980 \\
\hline $\mathrm{DH} 5 \alpha \mathrm{pSW}-2$ & $\begin{array}{l}\text { supE44, } \Delta \text { lacU169 (Ф80lacZ } \Delta \mathrm{M} 15), \text { hsdR17 }\left(\mathrm{r}_{K}-\mathrm{m}_{K}^{+}\right) \text {, recA1, endA1, thi-1, gyrA96, relA1; } \\
\text { harboring plasmid pSW-2 encoding I-Scel nuclease, tool for genomic deletion }\end{array}$ & $\begin{array}{l}\text { Martínez-García and de } \\
\text { Lorenzo, } 2011\end{array}$ \\
\hline PIR2 pEMG-fleQ & PIR2 harboring plasmid pEMG-fleQ & this study \\
\hline DH5 $\alpha \lambda$ pir pTNS1 & $\lambda$ pir lysogen of $\mathrm{DH} 5 \alpha$; harboring plasmid pTNS1 & Choi et al., 2005 \\
\hline DH5 $\alpha \lambda$ pir pSKO2 & $\begin{array}{l}\text { DH5 } \alpha \lambda \text { pir harboring Tn7 delivery vector pSK02 for chromosomal integration; containing } r h I A B \\
\text { genes from } P \text {. aeruginosa PA01 }\end{array}$ & Bator et al., 2020 \\
\hline \multicolumn{3}{|l|}{ P. putida } \\
\hline KT2440 & wild type & Bagdasarian et al., 1981 \\
\hline KT2440 E1 & Isolate of $P$. putida KT2440 after 23rd transfer from laboratory evolution on ethanol & This study \\
\hline KT2440 E1.1 & Isolate of $P$. putida KT2440 after 33rd transfer from laboratory evolution on ethanol & This study \\
\hline KT2440 $\Delta$ fleQ & $\Delta f l e Q$ & This study \\
\hline KT2440 SK4 & wild type with attTn7:Pffg $-r h / A B$ & Tiso et al., 2020 \\
\hline KT2440 E1.1_RL & KT2440 E1.1 with attTn7:Pffg - rhlAB & This study \\
\hline
\end{tabular}


of the cultures was centrifuged at $16,000 \times g$ for $2 \mathrm{~min}$, the supernatant was discarded, and the cells were resuspended in $1 \mathrm{~mL}$ RNAlater Stabilization Solution (Thermo Fisher Scientific, Waltham, MA, United States). All further steps for RNA extraction were performed using the Monarch Total RNA Miniprep Kit (New England Biolabs, Ipswich, MA, United States) following the manual. The RNA concentration was measured with the NanoDrop One (Thermo Fisher Scientific, Waltham, MA, United States) at $260 \mathrm{~nm}$. All samples were adjusted to a concentration of $6 \mathrm{ng} \mu \mathrm{L}^{-1}$ in a total volume of $40 \mu \mathrm{L}$. After RNA isolation, an additional DNase digestion was performed for $1 \mathrm{~h}$ at $37^{\circ} \mathrm{C}$ using $5 \mu \mathrm{L}$ DNase I and $5 \mu \mathrm{L}$ DNase I reaction buffer (New England Biolabs, Ipswich, MA, United States). The DNase I was inactivated at $75^{\circ} \mathrm{C}$ for $10 \mathrm{~min} .4 \mu \mathrm{L}$ of the RNA template were used for cDNA synthesis, which was performed using the ProtoScript II First Strand cDNA Synthesis Kit (New England Biolabs, Ipswich, MA, United States) following the manual. Subsequently, PCR was performed in white 96-well plates using the Universal qPCR Master Mix (New England Biolabs, Ipswich, MA, United States) in a CFX Connect Real-Time PCR Detection System (Bio-Rad Laboratories, Hercules, CA, United States). For qRT-PCR, specific oligonucleotides with annealing temperatures of $60^{\circ} \mathrm{C}$ were designed using the Primer3 software ${ }^{1}$. Standard curves for all primer pairs were performed by using two-fold dilution series of genomic DNA of the wild type with seven data points. Afterward, the primer efficiencies were determined and reached values between 2 and 2.13. Nuclease-free water for every primer pair and DNase treated RNA templates were used as negative controls. Each cDNA sample was diluted 1:10 and analyzed as technical triplicate. Data analysis was performed using the Bio-Rad CFX Manager Software. The resulting Ct values were used to calculate the gene expression level via the $2^{\Delta \mathrm{Ct}}$ method by normalizing to the house keeping gene $r p o B$.

For the deletion of the fleQ gene (PP_4373) the I-SceI-based system developed by Martínez-García and de Lorenzo (2011) was used. Briefly, $500 \mathrm{bp}$ upstream and downstream flanking regions were amplified from the genomic DNA of $P$. putida KT2440 with the Q5 High-Fidelity polymerase (using primers IB-278, IB-279, IB-280, and IB-281) and cloned into the nonreplicative pEMG vector by Gibson Assembly (Gibson et al., 2009). All used primers with their nucleotide sequences are listed in the Supplementary Material (Supplementary Table 1). The resulting plasmid pEMG-fleQ was transferred into chemically competent E. coli PIR2 cells by heat shock (Hanahan, 1983). The plasmids were isolated using the Monarch Plasmid Miniprep Kit (New England Biolabs, Ipswich, MA, United States) and validated by Sanger sequencing performed by Eurofins Genomics (Ebersberg, Germany). Afterward, the plasmids were transferred to $P$. putida KT2440 via conjugation. Triparental mating using a streamlined method as outlined by Wynands et al. (2018) was performed. After mating procedures, $P$. putida strains were selected on cetrimide agar (Sigma-Aldrich, St. Louis, MO, United States). For restriction, plasmid pSW-2 was used and no 3-methylbenzoate for induction of I-SceI expression was required. Deletion mutants were verified by colony PCR using

${ }^{1}$ http://primer3.ut.ee
OneTaq 2x Master Mix with Standard Buffer and primers IB-282 and IB-283. At last, the recombinant strains were cured of pSW-2 plasmid by re-inoculation in LB medium without antibiotics.

To obtain biosurfactant (HAA and mono-rhamnolipid) producing strains, the mini-Tn7 delivery transposon vector developed by Zobel et al. (2015) was used. The biosurfactant synthesis module is inserted in a single genomic locus of the chromosome (att $\operatorname{Tn} 7)$. The plasmid pSK02 harboring the $\operatorname{rhlAB}$ genes from $P$. aeruginosa PA01 was integrated into the genome of $P$. putida KT2440 E1.1 via transposition. Identification and selection of biosurfactant producing clones was performed as described by Bator et al. (2020), using ethanol as substrate instead of glucose.

\section{Analytical Methods Analysis of Bacterial Growth}

Bacterial growth was determined by measuring the optical density at $600 \mathrm{~nm}\left(\mathrm{OD}_{600}\right)$ with the Ultrospec 10 cell density meter (Biochrom, Cambridge, United Kingdom). A correlation between $\mathrm{OD}_{600}$ and cell dry weight $(\mathrm{CDW})$ was generated. An $\mathrm{OD}_{600}$ of 1.0 corresponds with a CDW of $313 \mathrm{mg} \mathrm{L}^{-1}$.

\section{Analysis of Metabolites and Products}

Sample preparation and analysis via high performance liquid chromatography (HPLC) for the measurement of ethanol and extracellular metabolites, e.g., acetate, was performed with the same method for glucose and other organic acids as described in Bator et al. (2020). It was used a Metab-AAC $300 \times$ $7.8 \mathrm{~mm}$ separation column (particle size: $10 \mu \mathrm{m}$, ISERA GmbH, Düren, Germany). For the measurement of HAAs and monorhamnolipids, sample preparation and analysis via HPLC was performed according to Bator et al. (2020) using a NUCLEODUR C18 Gravity $150 \times 4.6 \mathrm{~mm}$ separation column (particle size: $3 \mu \mathrm{m}$, Macherey-Nagel GmbH \& Co., KG, Düren, Germany).

\section{Fermentation Conditions}

Fed-batch fermentations for rhamnolipid production were performed as duplicate using modified M9 minimal medium with a final composition (per L) of $1.21 \mathrm{~g} \mathrm{Na}_{2} \mathrm{HPO}_{4} \cdot 2 \mathrm{H}_{2} \mathrm{O}, 0.43 \mathrm{~g}$ $\mathrm{KH}_{2} \mathrm{PO}_{4}, 0.5 \mathrm{~g} \mathrm{NaCl}, 1.9 \mathrm{~g} \mathrm{NH}_{4} \mathrm{Cl}, 2 \mathrm{mM} \mathrm{MgSO} 4,9.74 \mathrm{mg}$ $\mathrm{FeSO}_{4} \cdot 7 \mathrm{H}_{2} \mathrm{O}, 41.2 \mathrm{mg} \mathrm{CaCl}_{2} \cdot 2 \mathrm{H}_{2} \mathrm{O}, 1.5 \mathrm{mg} \mathrm{MnCl}_{2} \cdot 4 \mathrm{H}_{2} \mathrm{O}$, $1.87 \mathrm{mg} \mathrm{ZnSO} \cdot \cdot 7 \mathrm{H}_{2} \mathrm{O}, 0.3 \mathrm{mg} \mathrm{H}_{3} \mathrm{BO}_{3}, 0.25 \mathrm{mg} \mathrm{Na} \mathrm{MoO}_{4} \cdot 2 \mathrm{H}_{2} \mathrm{O}$, $0.15 \mathrm{mg} \mathrm{CuCl} 2 \cdot 2 \mathrm{H}_{2} \mathrm{O}, 13.11 \mathrm{mg} \mathrm{Na} \mathrm{N}_{2}$ EDTA $2 \mathrm{H}_{2} \mathrm{O}$ (Müller et al., personal communication), and $7.6 \mathrm{~g}$ ethanol $(0.33 \mathrm{Cmol})$ for the batch phase. The fermentations were conducted in a stirred tank reactor (BioFlo 120 glass bioreactor; Eppendorf, Hamburg, Germany) with a working volume of $1 \mathrm{~L}$ and a nominal volume of 1.3 L. The fermentations were controlled by a BioFlo120 unit and DASware control software (v5.5.0; Eppendorf, Hamburg, Germany). The setup is illustrated in Figure 1. The bioreactor was equipped with a pH electrode (EasyFerm plus PHI 225, Hamilton, Reno, NV, United States), dissolved oxygen (DO) electrode (InPro 6800, Mettler-Toledo, Columbus, OH, United States), sparger, $\mathrm{pH}$ control agent inlets, foam outlet, foam recirculation inlet, septum, Pt100 temperature sensor, hose connection for the inoculation bottle, sampling system, and cooling loop. The agitator shaft was equipped with two Rushton turbines ( $\varnothing$ 


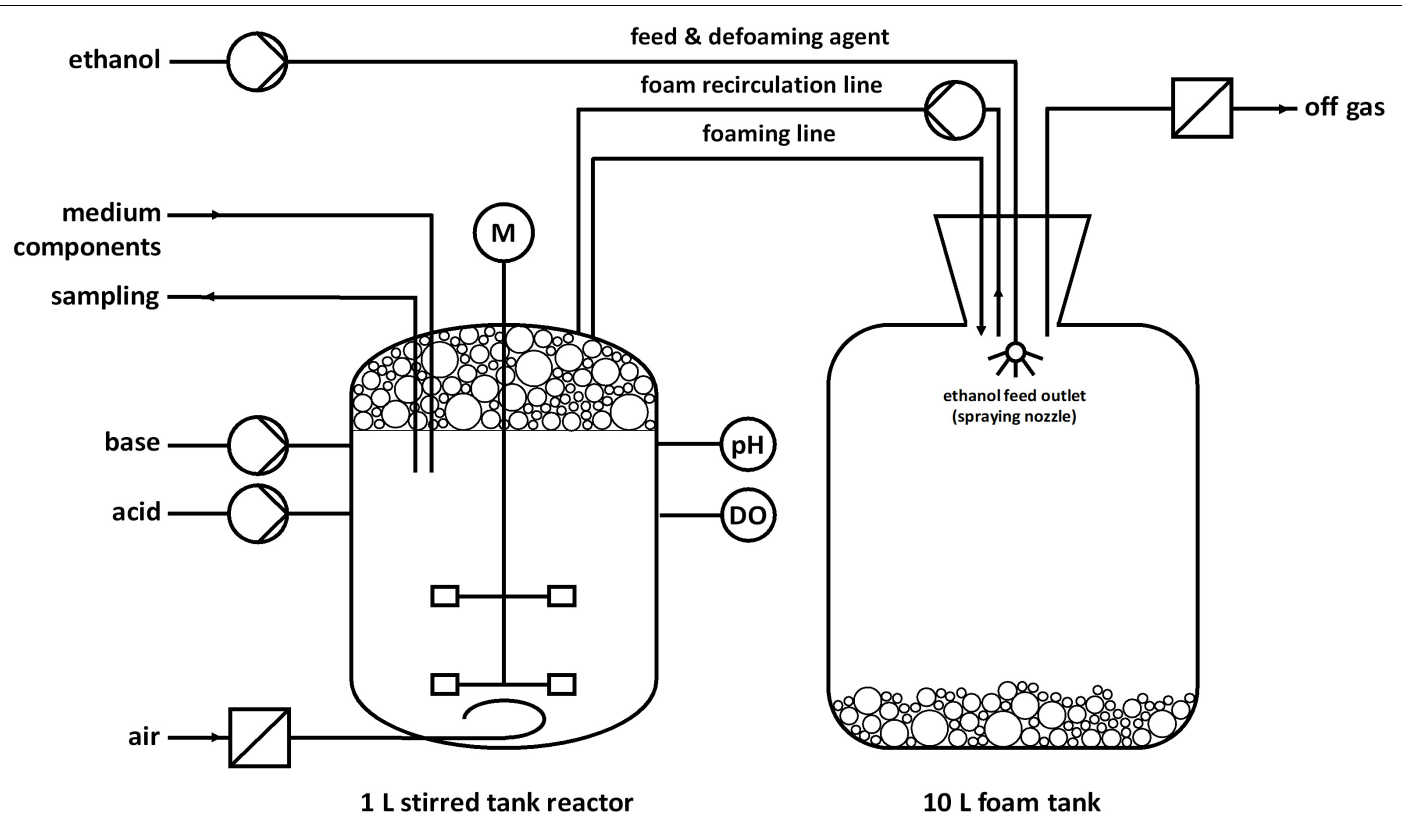

FIGURE 1 | Schematic setup for a fed-batch fermentation in a bioreactor with foam recirculation and pure ethanol as feed and defoaming agent. Foam is produced during the fermentation process and collected in a foam tank. Ethanol was automatically sprayed inside the foam tank based on a DO-based feeding protocol. After the addition of ethanol, the collected foam collapsed in the tank and was continuously pumped back as a liquid.

$5.3 \mathrm{~cm}$ ). The temperature was held constant at $30^{\circ} \mathrm{C}$ and the $\mathrm{pH}$ was adjusted to 7 via automatic addition of $30 \% \mathrm{NH}_{4} \mathrm{OH}$ and $2 \mathrm{M} \mathrm{H}_{2} \mathrm{SO}_{4}$. $\mathrm{NH}_{4} \mathrm{OH}$ simultaneously served as nitrogen source. The aeration rate was $0.1 \mathrm{~L} \mathrm{~min}^{-1}(0.1 \mathrm{vvm})$, whereas evaporation was reduced by sparging the supply of sterile air through a sterile water bottle. The DO was kept above $30 \%$ by an automatic increase of the agitation speed until 1,200 rpm. The foam produced during the fermentation was directed through the foam outlet via a hose into a downstream foam collection bottle. The reactor was inoculated with cells from a preculture to a final $\mathrm{OD}_{600}$ of 0.5 . One hour after inoculation, the peristaltic pump for foam recirculation was turned on at $220 \mathrm{rpm}(\varnothing$ of the inner hose $=1 \mathrm{~cm}$ ) and the stirrer speed was manually increased from 300 to $500 \mathrm{rpm}$ to obtain wet foam. After the batch phase, the feeding phase was initiated. The feed was realized with an automated DO-based feeding strategy via a peristaltic pump. The feed contained 100\% (v/v) ethanol (Th. Geyer GmbH \& Co. KG, Renningen, Germany) and was activated at DO-levels higher than $40 \%$ and deactivated at DO-levels lower than 30\%. In one feeding event, $3 \mathrm{~mL}$ ethanol were pumped to avoid toxic concentrations. However, the feed did not enter the reactor directly but was sprayed into the foam tank to collapse the collected foam. The collapsed foam is pumped back into the reactor by a peristaltic pump. The stirrer speed was constant at 1,200 rpm and up to $90 \%$ pure oxygen was manually mixed to the supply air. When the growth rate decreased, medium components were added to maintain growth and avoid nutrition limitation at higher cell densities. At $20 \mathrm{~h}$ the last supplementation was done and the medium contained in total (per L) $7.2 \mathrm{~g} \mathrm{Na}_{2} \mathrm{HPO}_{4} \cdot 2 \mathrm{H}_{2} \mathrm{O}$, $2.58 \mathrm{~g} \mathrm{KH}_{2} \mathrm{PO}_{4}, 0.5 \mathrm{~g} \mathrm{NaCl}, 1.9 \mathrm{~g} \mathrm{NH}_{4} \mathrm{Cl}$, $9.51 \mathrm{~g} \mathrm{NH} \mathrm{NH}_{4} \mathrm{OH}$, $26 \mathrm{mM} \mathrm{MgSO}, 48.78 \mathrm{mg} \mathrm{FeSO}_{4} \cdot 7 \mathrm{H}_{2} \mathrm{O}, 0.22 \mathrm{~g} \mathrm{CaCl}_{2} \cdot 2 \mathrm{H}_{2} \mathrm{O}$,
$48.7 \mathrm{mg} \mathrm{MnCl}_{2} \cdot 4 \mathrm{H}_{2} \mathrm{O}, 10.85 \mathrm{mg} \mathrm{ZnSO}_{4} \cdot 7 \mathrm{H}_{2} \mathrm{O}, 3.37 \mathrm{mg} \mathrm{H} \mathrm{BO}_{3}$, $1.55 \mathrm{mg} \mathrm{Na} \mathrm{MoO}_{4} \cdot 2 \mathrm{H}_{2} \mathrm{O}, 1 \mathrm{mg} \mathrm{CuCl}_{2} \cdot 2 \mathrm{H}_{2} \mathrm{O}$, and $65.7 \mathrm{mg}$ $\mathrm{Na}_{2}$ EDTA. $2 \mathrm{H}_{2} \mathrm{O}$.

To characterize the fed-batch fermentation results, the values of biomass, biosurfactants, and ethanol (during batch phase) were fitted using a logistic equation with four parameters as described by Wittgens et al. (2011). An independent fit was used to illustrate the development of all three fermentation parameters.

\section{Flux Balance Analysis}

Flux balance analysis (FBA) has been carried out as described previously by Bator et al. (2020). Briefly, the genome-scale model of P. putida KT2440, iJN1463 (Nogales et al., 2020), was used and extended by the biosynthesis route for monorhamnolipid production. All simulations were carried out in MATLAB (version R2019b; The MathWorks, Inc., Natick, MA, United States). The uptake of ethanol was set to $10 \mathrm{mmol} \mathrm{g}_{C D W}{ }^{-1} \mathrm{~h}^{-1}$ and the uptake of other carbon sources (e.g., glucose) was set to zero. The maximization of production of mono-rhamnolipids or biomass was used as objective function in the extended model.

\section{RESULTS}

\section{Evolutionary Engineering Improves Growth of $P$. putida KT2440 on Ethanol}

In order to establish a rhamnolipid production process based on ethanol, the growth behavior of $P$. putida KT2440 was investigated in minimal medium with $0.96,2,4,6$, and $8 \%(\mathrm{v} / \mathrm{v})$ ethanol as sole carbon source. $0.96 \%$ (v/v) ethanol was included, 
since this equals $0.33 \mathrm{Cmol}$, which is the carbon content used in all further experiments in this study. Interestingly, the wild type was able to grow natively in medium containing up to $4 \%$ (v/v) ethanol (Figure 2B). However, cell clumping was observed, indicating cell stress. The wild type exhibits a long lag phase of about $70 \mathrm{~h}$ at $4 \%(\mathrm{v} / \mathrm{v})$ ethanol (Figure 2B), which is likely caused by the inhibitory effect of the high ethanol concentration. While even at $0.96 \%(\mathrm{v} / \mathrm{v})$ cell clumping was observed, the lag phase was significantly reduced to $6 \mathrm{~h}$ and a growth rate of $0.21 \pm 0.03 \mathrm{~h}^{-1}$ was reached (Figure 2A). However, the growth rate showed a sharp drop after approximately $16 \mathrm{~h}$. In general, the results show that $P$. putida KT2440 is able to utilize ethanol, which was not surprising since an ethanol oxidation system and several alcohol and aldehyde dehydrogenases exist in Pseudomonas species (Nelson et al., 2002; Görisch, 2003).

Because the cells clumped and had a moderate growth rate, we decided to improve these traits by ALE. ALE is a technique frequently used to improve features of microbes by adaptation to various environmental conditions, such as temperatures or non-preferred carbon sources. A first ALE experiment was performed using M9 minimal medium with $2 \mathrm{~g} \mathrm{~L}^{-1}$ glucose and $4 \%(\mathrm{v} / \mathrm{v})$ ethanol to increase the solvent tolerance for 30 days corresponding to $\sim 115$ generations. Single colonies were obtained from the inhomogeneous culture, which were tested for growth (data not shown). The best-adapted colony (the strain with the highest growth rate and shortest lag phase after ALE) was designated $P$. putida KT2440 E1, which was used for a second ALE experiment in M9 minimal medium with 4\% (v/v) ethanol for twelve days corresponding to $\sim 37$ generations. Again, single colony isolation, and growth rate determination was used; the best adapted strain was designated P. putida KT2440 E1.1. After ALE, both strains showed reduced or no cell clumping in the presence of ethanol, which might correlate with ethanol tolerance. The growth of the two adapted strains was investigated in M9 minimal medium containing 0.96 or $4 \%(\mathrm{v} / \mathrm{v})$ ethanol (Figures 2A,B). On 4\% (v/v) ethanol, a significantly decreased lag phase of 9 and $6 \mathrm{~h}$ was observed for P. putida KT2440 E1 and $P$. putida KT2440 E1.1, respectively (Figure 2B). P. putida KT2440 E1 had a slightly reduced growth rate of $0.17 \pm 0.02 \mathrm{~h}^{-1}$, while no drop of the growth rate when compared to the wild type in $0.96 \%(\mathrm{v} / \mathrm{v})$ ethanol was observed. The growth rate of $P$. putida KT2440 E1.1 was increased to $0.51 \pm 0.03 \mathrm{~h}^{-1}$.

Additionally, the formation of the intermediate acetate was examined. The intermediate acetaldehyde could not be measured due to its volatile character. The enzymatic steps and introduction in the central carbon metabolism are shown in Figure 3. Samples taken until $28 \mathrm{~h}$ of the cultivation of $P$. putida KT2440 using $0.96 \%(\mathrm{v} / \mathrm{v})$ ethanol could exclude the presence of acetate. Hence, it is hypothesized that the conversion of acetaldehyde to acetate is the rate-limiting step and instead, an acetaldehyde accumulation took place, which caused growth inhibition (drop of the growth rate after $16 \mathrm{~h}$ in Figure 2A) because aldehydes are highly toxic also at low levels. The evolved strains P. putida KT2440 E1 and E1.1 grew to higher cell densities, but they also showed higher acetate concentrations (3.1 \pm 0.3 and $\left.3.8 \pm 0.1 \mathrm{~g} \mathrm{~L}^{-1}\right)$, respectively. Due to ALE, the overall concentrations of the intermediates were not growthinhibiting anymore. As a consequence, the evolved strains were able to use ethanol and acetaldehyde more efficiently indicated by the higher biomass production. However, another imbalance was revealed after ALE, which resulted in a stronger acetate accumulation through a slow conversion of acetate to acetylCoA. Here, a higher growth rate on ethanol and a higher ethanol tolerance were achieved. The time period of the ALE process might be extended in order to select isolates with even more improved features, i.e., a higher growth rate and lower accumulation of intermediates. However, a short time period was chosen to minimize the appearance of random background mutations, simplifying the allocation of mutations to the observed phenotypes.

\section{Rerouting of Metabolism for Carbon-Efficient Ethanol Utilization Caused by Adaptation}

To investigate the basis of the phenotypic changes after ALE, genome re-sequencing of both evolved strains and $P$. putida KT2440 as reference was performed. Furthermore, the metabolic
A

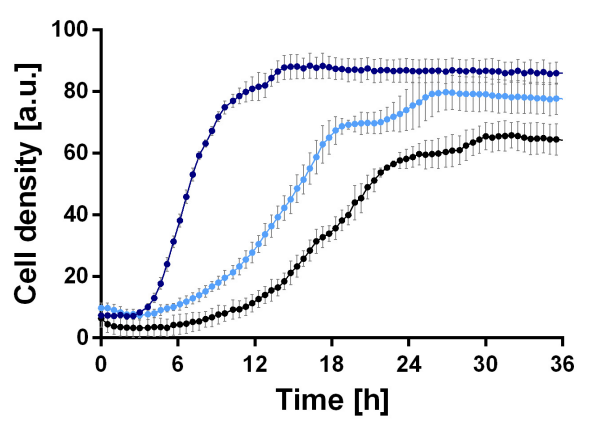

B

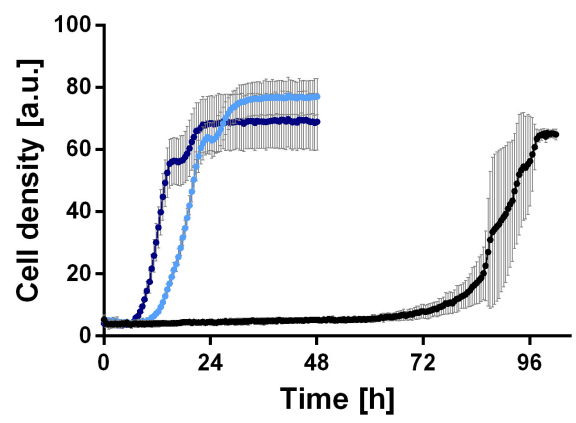

FIGURE 2 | Comparison of growth of P. putida KT2440 (black) and two evolved strains, P. putida KT2440 E1 (light blue) and P. putida KT2440 E1.1 (dark blue), in M9 minimal medium containing ethanol. (A) Growth in medium containing $0.96 \%(v / v)$ ethanol and (B) growth in medium containing $4 \%(v / v)$ ethanol. Growth was detected using a Growth Profiler 960 in 96-well plates. Error bars indicate the standard deviation from the mean $(n=3)$. 


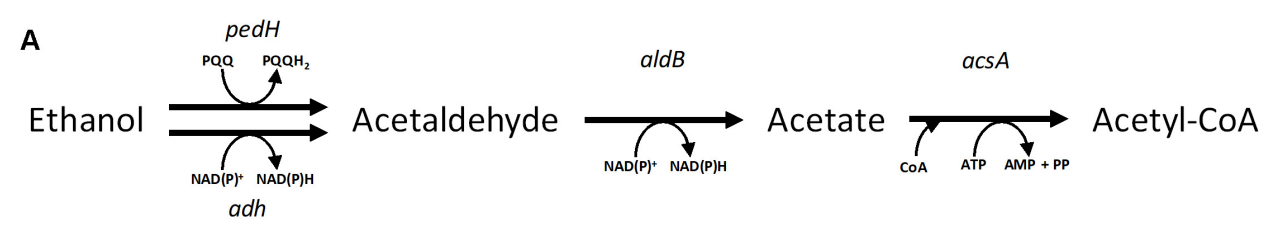

B

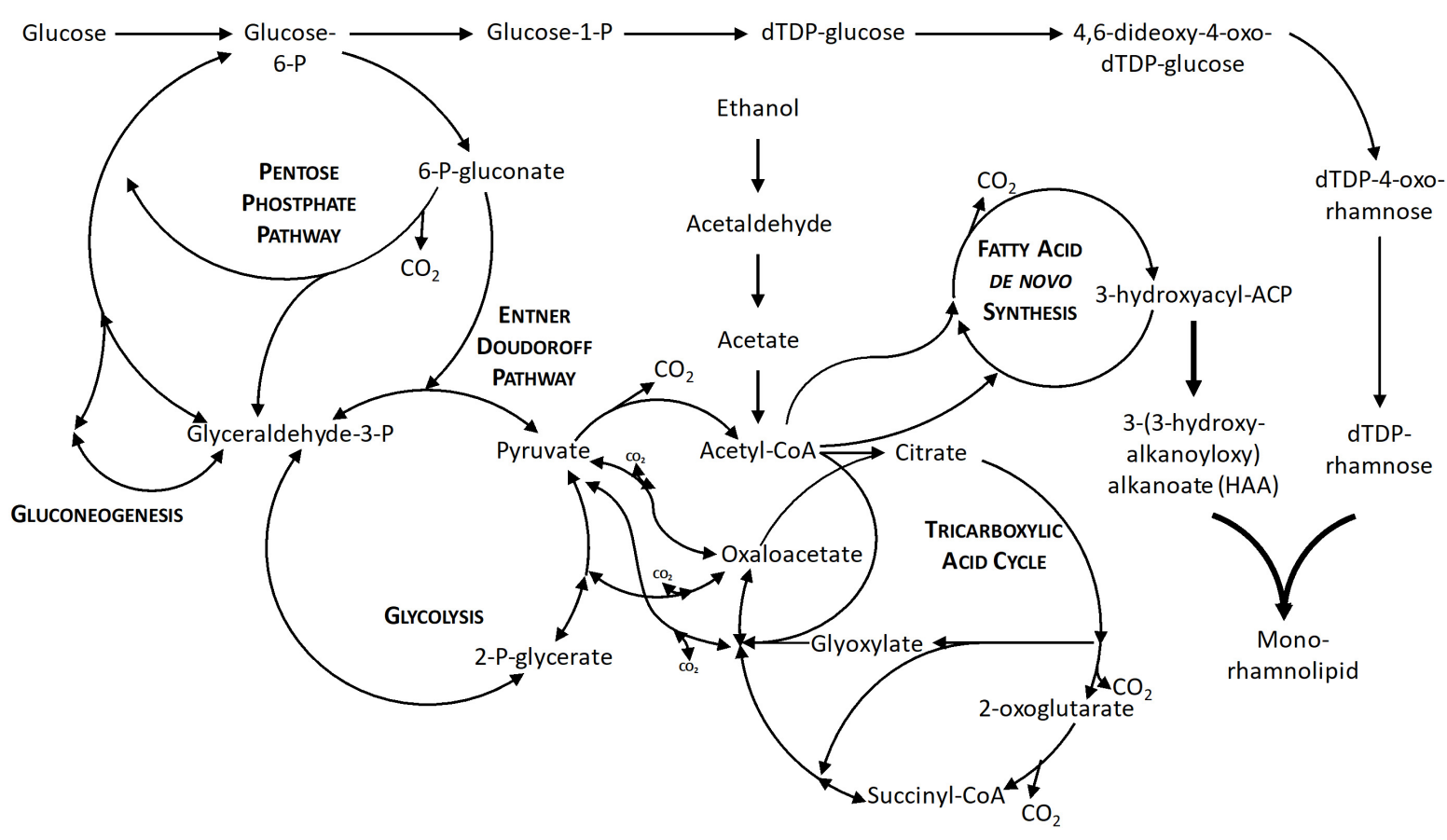

FIGURE 3 | The metabolic network of $P$. putida KT2440. (A) Enzymatic steps of ethanol utilization. (B) Central carbon metabolism of $P$. putida KT2440 based on Sudarsan et al. (2014) with the native ethanol utilization pathway and the heterologous mono-rhamnolipid production pathway. Bold arrows indicate the recombinant enzymatic steps toward mono-rhamnolipid production. pedH, quinoprotein ethanol dehydrogenase; adh, alcohol dehydrogenase; aldB, aldehyde dehydrogenase; acsA, acetyl-CoA synthetase; AMP, adenosine monophosphate; ADP, adenosine diphosphate; ATP, adenosine triphosphate; NAD ${ }^{+}$, nicotinamide adenine dinucleotide; $\mathrm{NADH}$, reduced nicotinamide adenine dinucleotide; $\mathrm{PQQ}$, pyrroloquinoline quinone; $\mathrm{PQQH}$, reduced pyrroloquinoline quinone.

network structure was examined by FBA. We could identify 60 mutations, including 36 SNPs and 24 insertion-deletion polymorphisms (InDel) for the reference strain $P$. putida KT2440 from our stain collection compared to the genome sequence of P. putida KT2440 (AE015451.2; Belda et al., 2016). This phenomenon was observed before in other P. putida KT2440 genome re-sequencing studies (Belda et al., 2016; Li et al., 2019). Similar mutation patterns of two reference strains from different laboratories were observed, even though the reference strains share no common history (Li et al., 2019). Most of the mutations are located in non-coding regions, silent mutations, or errors due to low coverage and read quality. Seven additional SNPs and one InDel were identified for the evolved E1. P. putida KT2440 E1.1 carried three SNPs and six InDels in addition to E1. Four genomic regions, which probably have an influence on ethanol tolerance and utilization were identified (Figure 4). More specifically, all SNPs and InDels of strains E1 and E1.1 were considered except silent mutations, mutations in hypothetical proteins, mutations with low coverage and read quality, and mutations in non-coding regions (except one mutation in a probable promoter region). Three mutations were found in strain E1 and three additional mutations in strain E1.1 (Supplementary Table 2). Two physiological effects can be distinguished: enhanced ethanol tolerance and more efficient ethanol utilization (higher ethanol uptake rate).

The mutations, which might effectuate a higher ethanol tolerance, are present in both strains and were thus introduced in the first evolution round (Figures 4B,D). These include a missense mutation in $s e c E$, which encodes a protein translocase subunit, and a nonsense mutation in $f l e Q$, which encodes a transcriptional regulator for flagellar and biofilm formation. The presence of organic solvents leads to altered lipid compositions and leakage of the plasma membrane in microbes as reviewed by Heipieper et al. (1994). The mutation in secE was perhaps introduced to counterbalance this effect due to an altered translocation of proteins across and insertion into the membrane. Furthermore, the nonsense mutation in fleQ likely causes the gene function to be negatively affected. In P. putida KT2440 
A

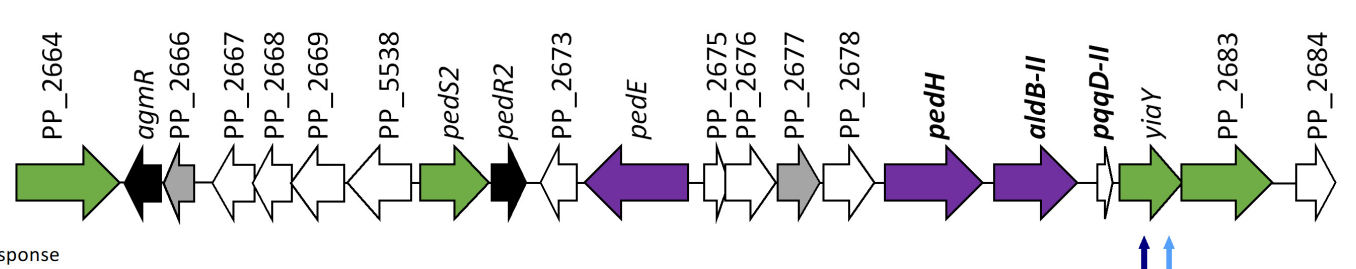

unknown response regulator

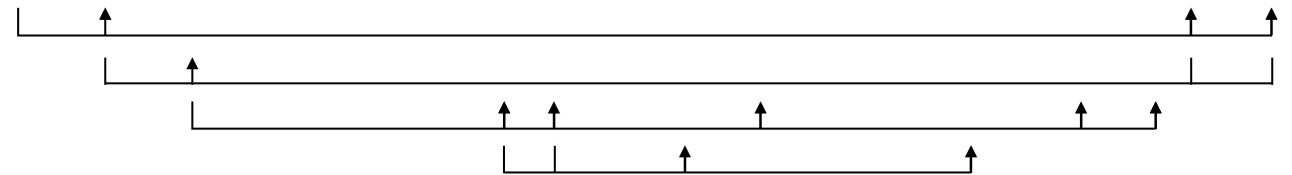

B

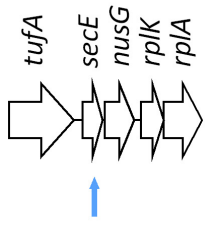

D
C

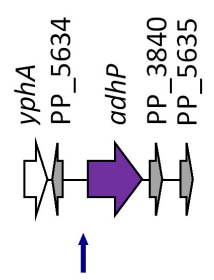

E

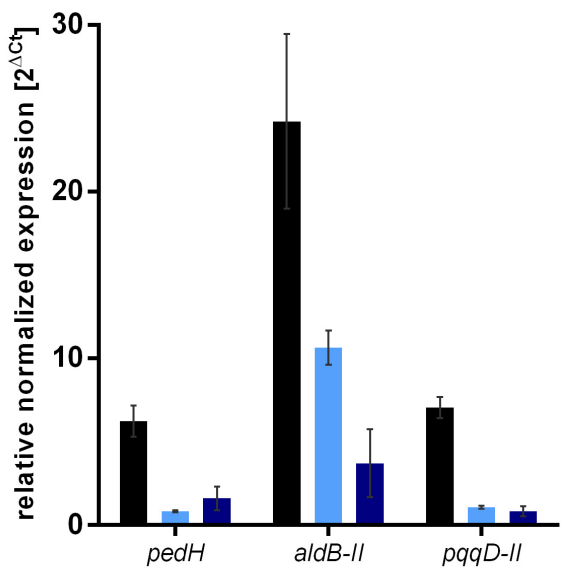

FIGURE 4 | Genomic consequences of ALE on gene expression. The gene organization is based on the genome sequence AE015451.2 (Belda et al., 2016). (A) Gene organization from PP_2664 to PP_2684 with a schematic hypothetical regulatory scheme below the genes based on regulation patterns in P. aeruginosa (Hempel et al., 2013). The black arrows display which genes have a positive regulatory influence on other genes. Further unidentified two-component systems genes are probably involved but not considered in this figure. The genes in bold font were investigated for gene expression levels. (B) Gene organization from tufA to rplA (PP_0440 to PP_0444). (C) Gene organization from yphA (PP_3837) to PP_5635. (D) Gene organization from fliE to fliS (PP_4370 to PP_4375). (E) Relative normalized expression of genes involved in ethanol metabolism of P. putida KT2440 (black bars), P. putida KT2440 E1 (light blue), and P. putida KT2440 E1.1 (dark blue) growing on $0.96 \%(\mathrm{v} / \mathrm{v})$ ethanol. The $2^{\Delta C t}$ values were normalized to rpoB and error bars represent the maximum and minimum values of two independent measurements $(n=2)$. Black arrows indicate genes coding for regulators, green arrows indicate genes coding for sensor kinases, purple arrows indicate genes involved in ethanol conversion, gray arrows indicate hypothetical proteins, and white arrows indicate genes with other functions. Smaller arrows below the gene organization show the identified mutations after ALE. The light blue arrows indicate the presence of the mutation in P. putida KT2440 E1 and the dark blue arrows indicate the presence of the mutation in P. putida KT2440 E1.1.

E1.1, a frameshift was additionally introduced in $f l e Q$ to abolish the gene function. In recent studies, it was reported that fleQdeficient $P$. putida strains showed a defect in swarming and reduced biofilm formation, which is caused by a lower expression of flagellar- and biofilm-related genes (Yousef-Coronado et al., 2008; López-Sánchez et al., 2016; Navarrete et al., 2019). Further, a relation between the disruption of flagellar-related genes and solvent tolerance in P. putida was described (DomínguezCuevas et al., 2006; Molina-Santiago et al., 2017). To verify this hypothesis, the gene fleQ was deleted. This deletion mutant, the reference strain, and the evolved strain E1.1 were then cultivated in minimal medium containing glucose and increasing ethanol concentrations. P. putida KT2440 $\Delta$ fleQ and P. putida KT2440
E1.1 showed an identical growth behavior when concentrations from 1 to $3 \%(\mathrm{v} / \mathrm{v})$ of ethanol were present (Figure 5). In contrast, the reference strain showed even in the presence of low ethanol concentrations a prolonged lag phase and decreased growth compared to the deletion mutant and the evolved strain (Figure 5). With the fleQ deletion we could show that i) the mutations in fle $Q$ are likely to contribute to an increased ethanol tolerance and ii) the mutations indeed in all likelihood lead to a deactivation of regulation of flagellar and biofilm formation.

Further mutations, which are likely assigned to ethanol utilization, were identified. Two mutations were found in a gene, of which the gene product is likely involved in the regulation of the ethanol oxidation system, which is encoded by genes localized 
A

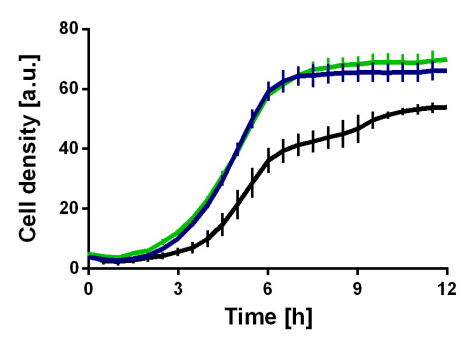

B

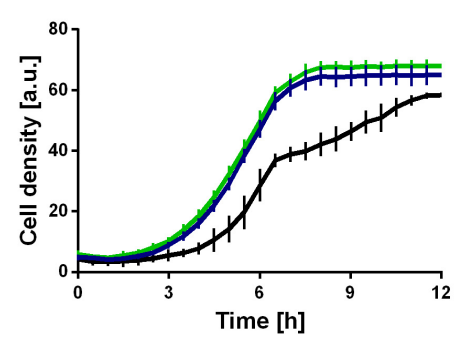

C

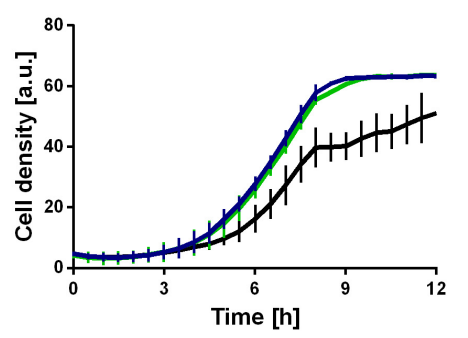

FIGURE 5 | Ethanol tolerance of P. putida KT2440 and derivatives in M9 minimal medium containing different ethanol concentrations. P. putida KT2440 (black), P. putida KT2440 E1.1 (blue), and P. putida KT2440 $\Delta$ fleQ (green). (A) Minimal medium containing $10 \mathrm{~g} \mathrm{~L}^{-1} \mathrm{glucose}$ and $1 \%$ (v/v) ethanol. (B) Minimal medium containing $10 \mathrm{~g} \mathrm{~L}^{-1}$ glucose and $2 \%$ (v/v) ethanol. (C) Minimal medium containing $10 \mathrm{~g} \mathrm{~L}^{-1} \mathrm{glucose}$ and $3 \%$ (v/v) ethanol. Growth was detected using a Growth Profiler 960 in 96-well plates. Error bars indicate the standard deviation from the mean $(n=3)$.

in an operon (Figure 4A) (Görisch, 2003; Belda et al., 2016). A missense mutation was introduced in yiaY (PP_2682) and in the longer evolved strain an additional nonsense mutation was introduced upstream in the same gene (Supplementary Table 2). The gene yiaY is proposed to encode a Fe-containing alcohol dehydrogenase. A genome analysis using BLAST (Altschul et al., 1990) showed that yiaY (PP_2682) and PP_2683 (a twocomponent system sensor histidine kinase/response regulator) of $P$. putida KT2440 have sequence similarities of $84 \%$ to $\operatorname{ercA}$ (PA1991) and $72 \%$ to ercS (PA1992) of P. aeruginosa PAO1, respectively. The gene ercA forms an operon with ercS and the gene product is presumed to generate a signal that activates the sensor kinase ErcS. This has an impact on the regulator AgmR, which controls the transcription of genes encoding components for ethanol oxidation (Figure 4A). Consequently, the gene product of ercA is part of a complex regulatory system and controls the expression of the quinoprotein ethanol oxidation genes in P. aeruginosa (Gliese et al., 2004; Hempel et al., 2013). Since the synteny of the ethanol oxidation system in P. putida and $P$. aeruginosa share similarities, we assumed that yiaY has a similar function in $P$. putida KT2440. To confirm this assumption, gene expression levels of the presumably regulated genes pedH, aldB-II, and pqqD-II, which are involved in ethanol oxidation (Figure 4A), in P. putida KT2440 and the two evolved strains E1 and E1.1 were determined via qRT-PCR. Interestingly, the transcript level of all three genes was highest for the reference strain (Figure 4E). P. putida KT2440 E1 had a lower transcript level of the regulated ethanol oxidation genes compared to the reference strain and the transcript levels of these genes of strain E1.1 were even lower. These findings indicate that yiaY is involved in the regulation of expression of ethanol oxidation genes since no other mutations were found in the surrounding kinases or regulators. Further, a low transcription of the genes involved in ethanol oxidation ( $p e d H$, aldB-II, and pqqD-II) could be observed in P. putida KT2440 E1.1, although a stop codon was introduced in yiaY. The stop codon was present in the second half of the gene and thus, there might be a residual activity. As mentioned before, the ethanol oxidation system is regulated by a complex regulatory system and other factors also might still regulate the transcription of the genes positively.
However, it was observed that the transcript levels of the ethanol oxidation genes in the operon were more decreased the longer the strain was evolved by ALE. This fits the hypothesis, which was formulated in the beginning based on the growth of the strains. It was argued that the overall concentration of the intermediates was not growth-inhibiting anymore and that an adaptation to the intermediates happened. These changes were the basis for more efficient use of the substrate. The gradual reduction of transcript identified by qRT-PCR entailed a slower conversion of ethanol and thus, lower concentrations of the toxic intermediate acetaldehyde might be present. Accumulation of acetaldehyde was suggested as a reason for the sharp drop of the growth rate for the wild type, which was not present in both evolved strains. In strain E1, acetaldehyde accumulation might be reduced below a toxic level for the cells enabling the further conversion of acetaldehyde into acetate. However, acetate accumulation was observed, indicating a second bottleneck (slow conversion to acetyl-CoA). This could have led to the further decrease of transcript levels, which is probably caused by the missense mutation in yia $Y$ in strain E1.1. The mutation has a reductive effect on the expression of the quinoprotein ethanol oxidation gene, pedH, and $a l d B-I I$ and possibly forces the strain to convert ethanol slower.

In P. putida KT2440 E1.1, the transcript levels of the genes for the ethanol oxidation enzymes, located in the operon (Figure 4E), were further decreased. However, other dehydrogenases may undertake the ethanol oxidation. One mutation in a gene, which is probably involved in ethanol degradation, occurred, causing that the encoded enzyme may undertake the conversion of ethanol to acetaldehyde. A SNP in the intergenic region before adhP (PP_3839), encoding an alcohol dehydrogenase, which is proposed to be located in the cytoplasm, was found (Figure 4C). The mutation upstream of $a d h P$ might alter the promoter activity upstream of adhP. Using the Softberry BPROM tool (Solovyev and Salamov, 2011), different promoters were predicted for the reference strain and $P$. putida KT2440 E1.1, i.e., the -10 region of the probable promoter upstream of adhP is affected by the mutation (Supplementary Figure 1). Thus, the alteration caused by the mutation might have contributed to a significantly improved growth rate (Figure 2). 
Although the mutations and resulting phenotypes, including the discovery of their underlying basis, were analyzed, the question why the quinoprotein dependent ethanol oxidation was downregulated and other dehydrogenases overtook their functions, are intriguing. To investigate this, FBA was used to predict fluxes and yields regarding biomass production using the genome-scale model P. putida KT2440 iJN1463 (Nogales et al., 2020). Two different scenarios, the PQQ-dependent and $\mathrm{NAD}^{+}$dependent ethanol conversion, were tested. Maximal product yields of $37 \mathrm{mmol} \mathrm{mol}^{-1}$ and $41 \mathrm{mmol} \mathrm{mol}^{-1}$ were computed for the PQQ-dependent ethanol conversion and the $\mathrm{NAD}^{+}$dependent ethanol conversion, respectively. To elucidate where the differences derive from, different fluxes were analyzed. In the reference strain P. putida KT2440 (PQQ-dependent), the electron pair is used in the respiratory chain for ATP synthesis and not for biomass production, thus a higher flux through the TCA cycle is needed to regenerate more NADH theoretically. With FBA, a higher respiration (14.7 mmol $\mathrm{g}_{C D W}{ }^{-1} \mathrm{~h}^{-1} \mathrm{O}_{2}$ ) and a higher flux through the TCA cycle, represented by the conversion of citrate to cis-aconitate $\left(4.87 \mathrm{mmol} \mathrm{g}_{C D W}{ }^{-1} \mathrm{~h}^{-1}\right)$, compared to the $\mathrm{NAD}^{+}$-dependent ethanol conversion was computed. This leads to a higher $\mathrm{CO}_{2}$ production $(5.33 \mathrm{mmol}$ $\mathrm{g}_{C D W}{ }^{-1} \mathrm{~h}^{-1}$ ) using the PQQ-dependent ethanol conversion instead of $3.52 \mathrm{mmol}_{\mathrm{CDW}}{ }^{-1} \mathrm{~h}^{-1} \mathrm{CO}_{2}$ using the $\mathrm{NAD}^{+}$dependent ethanol conversion. Thus, the changes obtained by ALE contributed to a more carbon-efficient route of ethanol degradation and led to an adaptation to the intermediates, which can explain the more efficient use of the substrate indicated by the higher biomass concentrations of the mutant strains E1 and E1.1.

\section{Biosurfactant Production Is Increased Due to More Efficient Substrate Utilization}

The production of mono-rhamnolipids and HAAs (from now on referred to as biosurfactants) was consequently investigated with an efficient ethanol metabolizer (P. putida KT2440 E1.1) and compared to the wild type in 24-deep well plates (Figure 6). For the production, the biosurfactant synthesis module was integrated as a single copy into the genome of $P$. putida KT2440 E1.1. The biosurfactant producer was designated $P$. putida KT2440 E1.1_RL. For comparison, the wild type with the integrated biosurfactant synthesis module, P. putida KT2440 SK4 (Tiso et al., in review), was used. The production was performed using $0.96 \%(\mathrm{v} / \mathrm{v})$ ethanol or $10 \mathrm{~g} \mathrm{~L}^{-1}$ glucose (both $0.33 \mathrm{Cmol} \mathrm{L}^{-1}$ ) as the sole carbon source to compare the production capacities on ethanol and the conventionally used glucose. The cultivation was performed for $48 \mathrm{~h}$ to ensure complete consumption of the substrates for both strains.

The product to substrate yield for P. putida KT2440 SK4 was in a similar range for glucose and ethanol, while the product to biomass yield was significantly higher (1.6-fold) when ethanol was used (Table 2). Further, the distribution of the different congeners was similar and the proportion of HAAs was in a similar range for both substrates (13 and 9\%), indicating that rhamnose provision is not significantly reduced when ethanol is used as a carbon source (Figure 6). The evolved strain E1.1

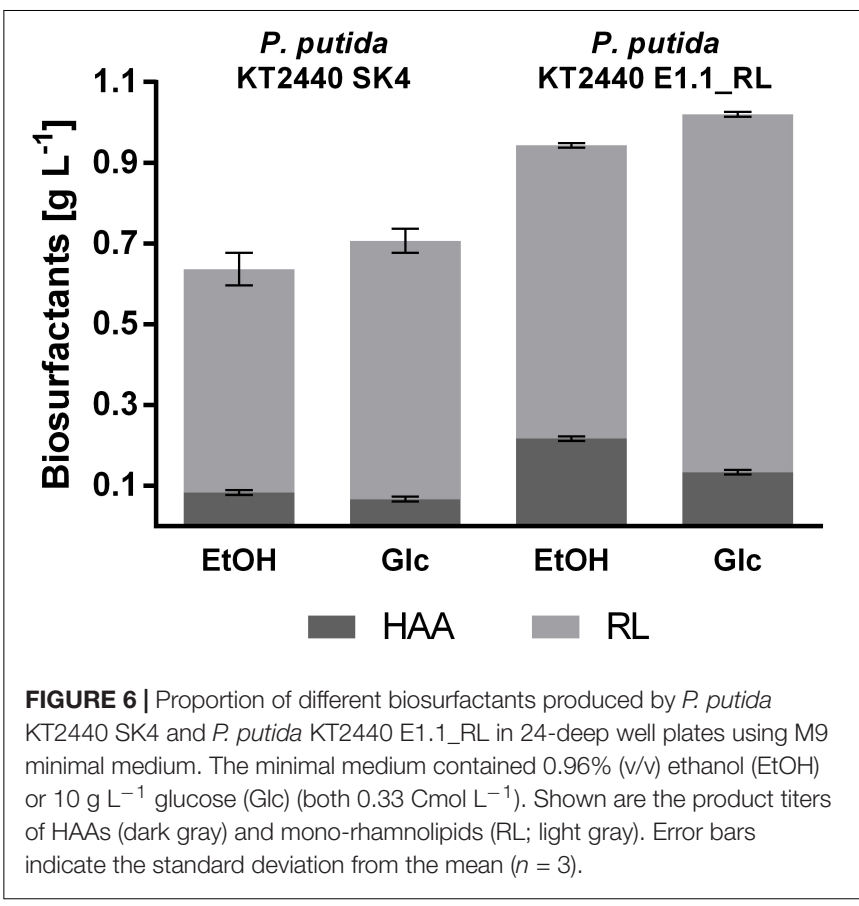

showed higher final biosurfactant titers, which is possibly caused by the higher biomass concentration. This also suggests that the evolved strain E1.1 uses the substrates more efficiently. Whereas the cause for the higher production on glucose remains unknown, in the case of ethanol, the rerouting of the metabolism is possibly the cause. As described before, the evolved strain downregulated the quinoprotein-dependent ethanol oxidation and instead likely used $\mathrm{NAD}^{+}$-dependent ethanol oxidation. With FBA not only for biomass production but also for rhamnolipid production, a higher yield using $\mathrm{NAD}^{+}$as cofactor was predicted. The predicted maximal product yield using PQQ and $\mathrm{NAD}^{+}$as a cofactor is 0.63 and $0.71 \mathrm{mmol} \mathrm{mmol}^{-1}$, respectively. Regarding the further evolved strain, E1.1 reached a similar product to substrate yield on ethanol compared to glucose but also reached a significantly higher product to biomass yield (Table 2). Further, the distribution of the different congeners was similar on both substrates, but a lower proportion of HAAs was produced on glucose (13\%) compared to ethanol (23\%) (Figure 6). This indicates a lack of activated rhamnose due to the conversion of ethanol to acetyl-CoA, which is then introduced in the tricarboxylic acid (TCA) cycle or the fatty acid de novo synthesis (Figure 3B). To obtain dTDP-L-rhamnose, the strain has to undertake gluconeogenesis to synthesize glucose-6-phosphate, which is energy-intensive. Since this phenomenon was only observed for P. putida KT2440 E1.1_RL, a reduced relative metabolic flux in the direction of hexoses after ALE is assumed yielding in a higher share of HAAs.

The product to biomass yield for each substrate is comparable for P. putida KT2440 SK4 and P. putida KT2440 E1.1_RL, with a higher product to biomass yield for ethanol. This points out that using ethanol leads to a higher production capacity per cell and that the stoichiometry of the ethanol degradation pathway 


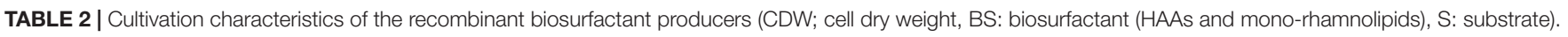

\begin{tabular}{|c|c|c|c|c|c|}
\hline Organism & Substrate & $\operatorname{CDW}\left(\mathrm{g}_{C D W} \mathrm{~L}^{-1}\right)$ & $\begin{array}{c}\text { Maximal titer } \\
\left(g_{B S} L^{-1}\right)\end{array}$ & $\begin{array}{l}\text { Product to substrate } \\
\text { yield }\left(\mathrm{Cmol}_{B S} \mathrm{Cmol}_{S}{ }^{-1}\right)\end{array}$ & $\begin{array}{l}\text { Product to biomass } \\
\text { yield }\left(g_{B S} g_{C D W}{ }^{-1}\right)\end{array}$ \\
\hline P. putida KT2440 SK4 & Ethanol $\left(7.6 \mathrm{~g} \mathrm{~L}^{-1}\right)$ & $1.11 \pm 0.08$ & $0.64 \pm 0.03$ & $0.10 \pm 0.00$ & $0.59 \pm 0.07$ \\
\hline P. putida KT2440 SK4 & Glucose $\left(10 \mathrm{~g} \mathrm{~L}^{-1}\right)$ & $1.94 \pm 0.03$ & $0.71 \pm 0.03$ & $0.11 \pm 0.00$ & $0.36 \pm 0.01$ \\
\hline P. putida KT2440 E1.1_RL & Ethanol $\left(7.6 \mathrm{~g} \mathrm{~L}^{-1}\right)$ & $1.63 \pm 0.05$ & $0.94 \pm 0.01$ & $0.15 \pm 0.00$ & $0.58 \pm 0.01$ \\
\hline P. putida KT2440 E1.1_RL & Glucose $\left(10 \mathrm{~g} \mathrm{~L}^{-1}\right)$ & $2.42 \pm 0.05$ & $1.02 \pm 0.01$ & $0.16 \pm 0.00$ & $0.42 \pm 0.01$ \\
\hline
\end{tabular}

is superior for biosurfactant production compared to glucose. Namely, ethanol is converted to acetyl-CoA directly being introduced in the fatty acid de novo synthesis without carbon loss, which is the precursor pathway for rhamnolipids (Figure 3B). Further, ethanol has a high degree of reduction, which means that more electrons are available and hence more energy is conserved in the substrate compared to glucose. This contributes to achieving a higher biomass or product yield. The theoretical product to substrate yield on ethanol is $0.93 \mathrm{Cmol} \mathrm{Cmol}^{-1}$ for zero growth, of which $16 \%$ were reached with $P$. putida KT2440 E1.1_RL. Further, the theoretical product to substrate yield on ethanol is $29 \%$ higher than on glucose.

\section{The Use of Ethanol Enables the Development of an Efficient Biosurfactant Production Process}

In order to increase the biosurfactant titer, a fed-batch fermentation procedure was developed. Biosurfactants tend to foam due to their amphiphilic structure. Thus, a fermentation process is challenging to control and often realized with additional equipment, chemicals, or process steps, which increases the costs and the effort in downstream processing. To keep the setup as simple as possible, an integrated foam recirculation with automated ethanol addition was developed.

The fermentations were conducted as biological duplicates with $P$. putida KT2440 E1.1_RL using ethanol as sole carbon source. In this setup, ethanol serves two functions. First, it is used as a carbon source for the production of biomass and biosurfactants. Second, it is used as a defoaming agent because the foam is destabilized when getting in contact with the ethanol. The collapsed foam with fresh carbon source was pumped back into the bioreactor, preventing thus loss of biomass and product while at the same time a fed-batch process is established.

The fermentation started with a batch phase $[0.96 \%(\mathrm{v} / \mathrm{v})$ ethanol], which ended after ethanol was consumed $(9.5 \mathrm{~h})$. A biosurfactant titer of about $0.7 \mathrm{~g} \mathrm{~L}^{-1}$ was reached after $9 \mathrm{~h}$ (Figure 7A). Further, a biomass concentration of around $3.5 \mathrm{~g} \mathrm{~L}^{-1}$ was reached (Figure 7A). The biosurfactant titer is $27 \%$ lower and the biomass concentration is 2-fold higher compared to the experiment in 24-deep well plates (Table 2). After the batch phase, the foam collection bottle was full of foam. To initiate the feed phase, $6 \mathrm{~mL}$ ethanol were added manually to collapse the foam. Subsequently, $3 \mathrm{~mL}$ ethanol were introduced automatically via the spraying nozzle by using a DO-based feeding script. The feed pulse was triggered when the DO signal increased to $40 \%$. To account for the built-in inertia of the control loop, which is schematically demonstrated in Figure 8A, a break of 5 min was
A
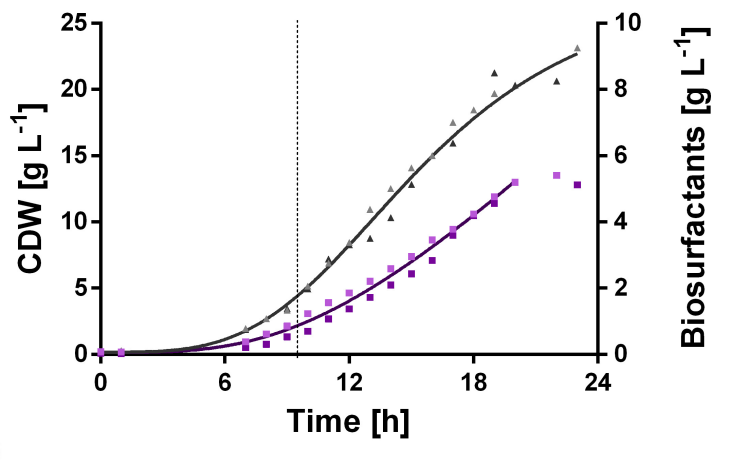

B

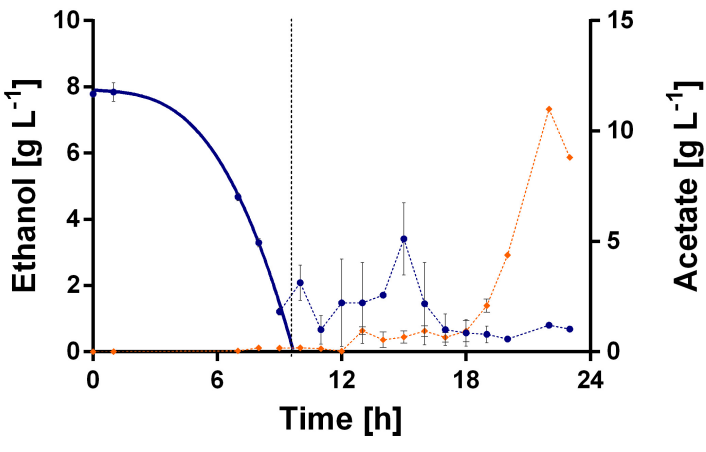

FIGURE 7 | DO-triggered fed-batch fermentation for biosurfactant production with P. putida KT2440 E1.1_RL using ethanol as sole carbon source. The fermentation was performed as a duplicate. (A) CDW ( $\mathbf{\Lambda}$, gray) and biosurfactant concentration ( $\mathbf{\square}$, purple) and their respective fitted courses. The values are given as single representative values of two bioreactor cultivations. (B) Ethanol concentration ( $\bullet$, blue) and acetate concentration ( $\downarrow$, orange) and the respective fitted course for ethanol in the batch phase. The dashed lines indicate the switch from batch to the fed-batch phase. The values are given as mean values of two bioreactor cultivations and error bars represent the maximum and minimum values of two independent experiments.

introduced after the feed trigger. During this pause, the DO signal dropped below $30 \%$, causing the script to cease feeding. Once the DO signal reached again $40 \%$, a new feed cycle was initialized, which can be seen in the oscillating DO signal (Figure 8B). In total, around $90 \mathrm{~mL}$ ethanol were fed. At the end of the fermentation a biosurfactant titer of about $5.3 \mathrm{~g} \mathrm{~L}^{-1}$ and biomass concentrations of about $22 \mathrm{~g} \mathrm{~L}^{-1}$ were reached. A growth rate of $0.14 \pm 0.00 \mathrm{~h}^{-1}$ was reached in the feed phase, which marks a reduction by $60 \%$ compared to the batch phase. This was likely caused by carbon limitation due to the linear feeding profile 


\section{A}

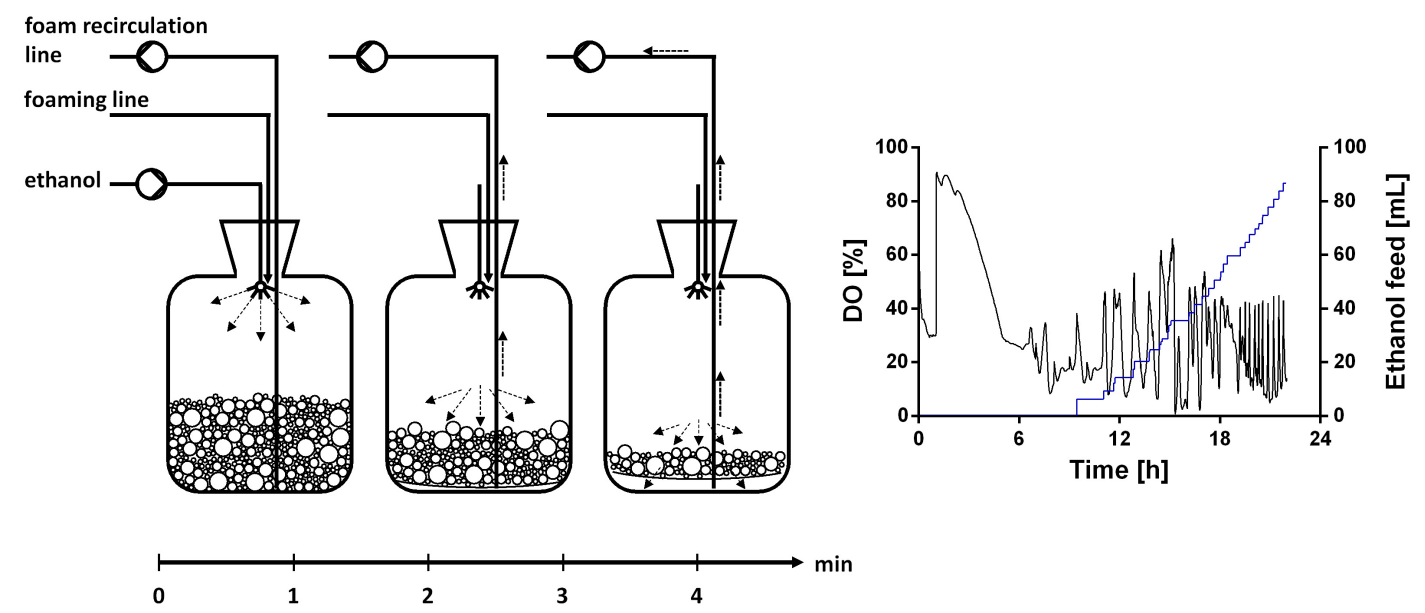

FIGURE 8 | Ethanol feeding procedure. (A) Schematic overview of the ethanol feed in the foam collection bottle based on a DO-based feeding protocol. (B) DO signal (black), which is controlled by a DO-based feeding protocol, and ethanol feed volume (blue), exemplarily shown for one reactor.

(Figure 8B). With this setup, a product to substrate yield of $0.065 \pm 0.006 \mathrm{~g}_{B S} \mathrm{~g}_{E t O H}{ }^{-1}$ was reached. The yield is $41 \%$ reduced compared to the production experiment in 24-deep well plates, which can be explained by the high biomass production, which benefits from the high oxygen and nitrogen supply. Further, a space time yield of $0.23 \pm 0.01 \mathrm{~g}_{B S} \mathrm{~L}^{-1} \mathrm{~h}^{-1}$ was achieved over the whole fermentation.

Again, acetate accumulation occurred after $18 \mathrm{~h}$ of fermentation. At the end of the fermentation an acetate concentration of about $10 \mathrm{~g} \mathrm{~L}^{-1}$ was observed. This entails two issues: On the one hand, the carbon source was thus not completely available for biosurfactant production, which adulterates the yield. Since the accumulated acetate can theoretically be converted to biosurfactants, the actual yield is $0.072 \pm 0.007 \mathrm{~g}_{B S} \mathrm{~g}_{E t O H}{ }^{-1}$. On the other hand, high acetate concentrations might cause growth inhibition. Contrary to the flask experiments of $P$. putida KT2440 E1.1, where approximately $3.8 \mathrm{~g} \mathrm{~L}^{-1}$ acetate accumulated, here no acetate was present after the batch phase. This might be caused by the introduction of additional metabolic demand for $\beta$-hydroxy fatty acids and thus acetyl-CoA (via biosurfactant production) and hence the increased conversion of acetate to acetyl-CoA.

This fermentation setup demonstrates the potential of using ethanol concomitantly as an efficient defoamer and carbon source. By automated ethanol addition, the supply of carbon is assured, resulting in an increase of biomass and biosurfactants throughout the whole fermentation. Additionally, no further equipment, such as mechanical foam breakers or antifoam, were necessary to control the foam.

Here, we show that the combination of strain and process design in an early stage resulted in the development of a novel fermentation setup. Strain engineering facilitated the use of ethanol, which subsequently could be used as foam-controlling agent and carbon source.

\section{DISCUSSION}

Taken together, the results show that a fed-batch rhamnolipid fermentation process can be developed without using conventional antifoam or mechanical foam breakers. To achieve this, we evolved $P$. putida KT2440 to an efficient ethanol metabolizer. The evolved strain was then characterized regarding growth and heterologous rhamnolipid production and was used to develop a unique rhamnolipid production process.

Considering the metabolic network configuration, ethanol is an ideal substrate for rhamnolipid production because it is converted to acetyl-CoA without carbon loss and feeds directly in the fatty acid de novo synthesis or the TCA cycle. Thereby, the formation of by-products derived from pyruvate can be circumvented and the yield may be increased. In addition, ethanol degradation delivers energy in form of NADH. This characteristic was already exploited for mevalonate production in $P$. putida KT2440 and rhamnolipid production in $P$. aeruginosa (Matsufuji et al., 1997; Santos et al., 2002; Yang et al., 2019). Moreover, bioethanol can be produced from alternative nonfood substrates, e.g., lignocellulosic biomass (Wendisch et al., 2016; Duwe et al., 2019). Thus, the use of ethanol as a carbon source contributes to establishing a circular bioeconomy while also creating an advantage in terms of developing a longlasting fermentation process. Ethanol has a defoaming property, which was described in a rhamnolipid production process using P. aeruginosa (Sha et al., 2012). While the ethanol has no defoaming or antifoaming effect in the fermentation broth, foam in a foam collection bottle collapses if ethanol is sprayed on it. Therefore, a setup with foam collection and recirculation was chosen (Figure 1). Sha et al. (2012) used a similar setup but used colza oil as substrate and manually added ethanol to collapse the collected foam. While the addition of ethanol before $48 \mathrm{~h}$ decreased the rhamnolipid and biomass production in their case, 
in our study, ethanol concomitantly served as carbon source and defoamer. The presence of ethanol to maintain growth and rhamnolipid production and collapsing of foam was ensured by a DO-based feeding script and hence occurred automatically. This setup theoretically allows the continuation of the fermentation beyond the batch phase for several days. Further, this study demonstrates the potential of combining strain and process development already in the early stages of process development. This integrated strain and process engineering approach was strongly emphasized by Kuhn et al. (2010), while consecutive optimization of strain and fermentation is still the norm. Engineering a biosurfactant producer that is able to tolerate high ethanol concentrations and utilize ethanol as carbon source unlocked the usage of the carbon source also as defoaming agent. The general strategy that the carbon source acts as antifoam is prominent in conventional rhamnolipid production using plant oils as carbon source. However, ethanol features the striking advantage of being water-soluble, which significantly reduces subsequent downstream processing efforts.

Compared to previous reports about recombinant rhamnolipid production with $P$. putida KT2440, the here obtained results using $P$. putida KT2440 as host for heterologous rhamnolipid production are comparable or even superior. In this fed-batch fermentation, a product to substrate yield of $0.072 \mathrm{~g}_{B S} \mathrm{~g}_{E t O H}{ }^{-1}$ and a space time yield of $0.23 \mathrm{~g}_{B S} \mathrm{~L}^{-1} \mathrm{~h}^{-1}$ was reached. In a study with an integrated rhamnolipid adsorption system using glucose as carbon source, a yield of $0.05 \mathrm{~g}_{R L} \mathrm{~g}^{-1}$ and a space time yield of $0.073 \mathrm{~g}_{R L} \mathrm{~L}^{-1} \mathrm{~h}^{-1}$ were reached (Anic et al., 2018). Beuker et al. (2016a) reported a titer of $14.7 \mathrm{~g} \mathrm{~L}^{-1}$, which corresponds to a yield of $0.09 \mathrm{~g}_{R L} \mathrm{~g}^{-1}$ and a space time yield of $0.2 \mathrm{~g}_{R L} \mathrm{~L} \mathrm{~h}^{-1}$ in a fed-batch fermentation using glucose and antifoam to control the foaming. With the native rhamnolipid producer $P$. aeruginosa a titer of $36.7 \mathrm{~g}_{R L} \mathrm{~L}^{-1}$ was achieved with a yield of $0.15 \mathrm{~g}_{R L} \mathrm{~g}^{-1}$ in a fermentation with foam separation using sunflower oil (Müller et al., 2011). While in general, hydrophobic substrates are known to generate the best product yields (Nitschke et al., 2011), also other carbon sources were already investigated. A fed-batch cultivation of $P$. aeruginosa using a medium supplemented with yeast extract and ethanol resulted in $32 \mathrm{~g}_{R L} \mathrm{~L}^{-1}$ rhamnolipids, which corresponds to a yield of $0.58 \mathrm{~g}_{R L} \mathrm{~g}^{-1}$ (Matsufuji et al., 1997). Hence, further improvements are needed to reach yields and titers comparable to those of $P$. aeruginosa with the here described setup.

Improvements envisaged are: First, a higher proportion of HAAs was produced by the evolved P. putida KT2440 E1.1_RL strain on ethanol (Figure 6), which indicates a bottleneck in the availability of the precursor dTDP-L-rhamnose. This might be diminished by the addition of hexoses as glucose, since glucose is directly used in the rhamnose pathway to yield dTDP-Lrhamnose (Rahim et al., 2000). Metabolic pathways for utilization of various substrates have natural stoichiometries leading to biosynthetic imbalance and suboptimal product yields (Park et al., 2019). Thus, using a mixture of substrates with introduction in several pathways may meet the requirements for optimal biosynthesis. However, some substrates are preferred and thus preventing the co-metabolization of less preferred substrates. This challenge was addressed by Park et al. with a synergistic substrate cofeeding approach, where a controlled limited feeding of glucose as dopant enabled a co-metabolization of acetate and glucose and enhanced product synthesis. Especially the use of least preferred carbon sources, such as $\mathrm{CO}_{2}$ or acetate, can be facilitated with such an approach contributing to a sustainable bioeconomy (Park et al., 2019). Second, acetate accumulation in the fed-batch fermentation after $18 \mathrm{~h}$ was observed (Figure 7B). A concentration of over $10 \mathrm{~g} \mathrm{~L}^{-1}$ acetate likely caused growth inhibition. Recently, Arnold et al. (2019) tested $10 \mathrm{~g} \mathrm{~L}^{-1}$ acetate as the sole carbon source for rhamnolipid production with a recombinant $P$. putida KT2440 and also identified a growth inhibition. Further, an acetate accumulation during growth on ethanol was reported for P. putida KT2440 (Yang et al., 2019). The authors hypothesized that the oxidation of ethanol to acetate is rapid and that the rate of acetate conversion to acetyl-CoA is limited and thus acetate accumulates. Yang et al. produced mevalonate using ethanol and observed acetate accumulation for a strain carrying the empty plasmid, while no acetate was present when the strain produced mevalonate. This corresponds with our results from batch cultivations, promoting our hypothesis that there is an additional metabolic demand for acetyl-CoA when thereof derived products are synthesized.

In summary, we report rhamnolipid production with a recombinant $P$. putida KT2440 derivative using ethanol as an alternative carbon source and defoamer, increasing substantially the possibilities in the fermentation set-up.

\section{DATA AVAILABILITY STATEMENT}

The raw data supporting the conclusions of this article will be made available by the authors, without undue reservation, to any qualified researcher.

\section{AUTHOR CONTRIBUTIONS}

IB performed all the engineering and characterization experiments, prepared the figures, and wrote the manuscript. TK performed the fermentations with the help of IB. TT and LB conceived the study, advised on all the experiments, discussed the data, and edited the manuscript. All authors contributed to the article and approved the submitted version.

\section{FUNDING}

The scientific activities of the Bioeconomy Science Center were financially supported by the Ministry of Culture and Science within the framework of the NRW Strategieprojekt BioSC (No. 313/323-400-00213). The laboratory of LB was partially funded by the Deutsche Forschungsgemeinschaft (DFG, German Research Foundation) under Germany's Excellence Strategy within the Cluster of Excellence FSC 2186 “The Fuel Science Center." 


\section{ACKNOWLEDGMENTS}

We kindly acknowledge Hans M. Böhne for his experimental help and thank Christian C. Blesken and Philipp Demling for all the discussions and support regarding fermentations.

\section{REFERENCES}

Altschul, S. F., Gish, W., Miller, W., Myers, E. W., and Lipman, D. J. (1990). Basic local alignment search tool. J. Mol. Biol. 215, 403-410. doi: 10.1016/S00222836(05)80360-2

Anic, I., Apolonia, I., Franco, P., and Wichmann, R. (2018). Production of rhamnolipids by integrated foam adsorption in a bioreactor system. AMB Express 8, 1-12. doi: 10.1186/s13568-0180651-y

Anic, I., Nath, A., Franco, P., and Wichmann, R. (2017). Foam adsorption as an ex situ capture step for surfactants produced by fermentation. J. Biotechnol. 258, 181-189. doi: 10.1016/j.jbiotec.2017.07.015

Arnold, S., Henkel, M., Wanger, J., Wittgens, A., Rosenau, F., and Hausmann, R. (2019). Heterologous rhamnolipid biosynthesis by P. putida KT2440 on bio-oil derived small organic acids and fractions. AMB Express 9, 1-7. doi: 10.1186/ s13568-019-0804-7

Bagdasarian, M., Lurz, R., Rückert, B., Franklin, F. C. H., Bagdasarian, M. M., Frey, J., et al. (1981). Specific-purpose plasmid cloning vectors II. Broad host range, high copy number, RSF 1010-derived vectors, and a host-vector system for gene cloning in Pseudomonas. Gene 16, 237-247. doi: 10.1016/0378-1119(81)90 080-9

Balan, V., Chiaramonti, D., and Kumar, S. (2013). Review of US and EU initiatives toward development, demonstration, and commercialization of lignocellulosic biofuels. Biofuels Bioprod. Bioref. 7, 732-759. doi: 10.1002/bbb. 1436

Banat, I. M., Franzetti, A., Gandolfi, I., Bestetti, G., Martinotti, M. G., Fracchia, L., et al. (2010). Microbial biosurfactants production, applications and future potential. Appl. Microbiol. Biotechnol. 87, 427-444. doi: 10.1007/s00253-0102589-0

Bator, I., Wittgens, A., Rosenau, F., Tiso, T., and Blank, L. M. (2020). Comparison of three xylose pathways in Pseudomonas putida KT2440 for the synthesis of valuable products. Front. Bioeng. Biotechnol. 7:480. doi: 10.3389/fbioe.2019. 00480

Belda, E., van Heck, R. G. A., José Lopez-Sanchez, M., Cruveiller, S., Barbe, V., Fraser, C., et al. (2016). The revisited genome of Pseudomonas putida KT2440 enlightens its value as a robust metabolic chassis. Environ. Microbiol. 18, 3403-3424. doi: 10.1111/1462-2920.13230

Bengelsdorf, F. R., and Dürre, P. (2017). Gas fermentation for commodity chemicals and fuels. Microb. Biotechnol. 10, 1167-1170. doi: 10.1111/17517915.12763

Beuker, J., Barth, T., Steier, A., Wittgens, A., Rosenau, F., Henkel, M., et al. (2016a). High titer heterologous rhamnolipid production. AMB Express 6, 1-7. doi: 10.1186/s13568-016-0298-5

Beuker, J., Steier, A., Wittgens, A., Rosenau, F., Henkel, M., and Hausmann, R. (2016b). Integrated foam fractionation for heterologous rhamnolipid production with recombinant Pseudomonas putida in a bioreactor. $A M B$ Express 6, 1-10. doi: 10.1186/s13568-016-0183-2

Beuker, J., Syldatk, C., and Hausmann, R. (2014). "Bioreactors for the production of biosurfactants," in Biosurfactants, eds N. Kosaric and F. Vardar-Sukan (Boca Raton, FL: CRC Press), 117-128. doi: 10.1201/b17599-10

Blank, L. M., Ebert, B. E., Buehler, K., and Bühler, B. (2010). Redox biocatalysis and metabolism: molecular mechanisms and metabolic network analysis. Antioxid. Redox Signal. 13, 349-394. doi: 10.1089/ars.2009.2931

Choi, K. H., Gaynor, J. B., White, K. G., Lopez, C., Bosio, C. M., KarkhoffSchweizer, R. A. R., et al. (2005). A Tn7-based broad-range bacterial cloning and expression system. Nat. Methods 2, 443-448. doi: 10.1038/nmeth765

Davis, D. A., Lynch, H. C., and Varley, J. (2001). The application of foaming for the recovery of surfactin from B. subtilis ATCC 21332 cultures. Enzyme Microb. Technol. 28, 346-354. doi: 10.1016/S0141-0229(00)00327-6

\section{SUPPLEMENTARY MATERIAL}

The Supplementary Material for this article can be found online at: https://www.frontiersin.org/articles/10.3389/fbioe. 2020.00899/full\#supplementary-material

Depristo, M. A., Banks, E., Poplin, R., Garimella, K. V., Maguire, J. R., Hartl, C., et al. (2011). A framework for variation discovery and genotyping using nextgeneration DNA sequencing data. Nat. Genet. 43, 491-498. doi: 10.1038/ng. 806

Desai, J. D., and Banat, I. M. (1997). Microbial production of surfactants and their commercial potential. Microbiol. Mol. Biol. Rev. 61, 47-64. doi: 10.1128/.61.1. 47-64.1997

Déziel, E., Lépine, F., Dennie, D., Boismenu, D., Mamer, O. A., and Villemur, R. (1999). Liquid chromatography/mass spectrometry analysis of mixtures of rhamnolipids produced by Pseudomonas aeruginosa strain 57RP grown on mannitol or naphthalene. Biochim. Biophys. Acta Mol. Cell Biol. Lipids 1440, 244-252. doi: 10.1016/S1388-1981(99)00129-8

Déziel, E., Lépine, F., Milot, S., and Villemur, R. (2000). Mass spectrometry monitoring of rhamnolipids from a growing culture of Pseudomonas aeruginosa strain 57RP. Biochim. Biophys. Acta Mol. Cell Biol. Lipids 1485, 145-152. doi: 10.1016/S1388-1981(00)00039-1

Déziel, E., Lépine, F., Milot, S., and Villemur, R. (2003). rhlA is required for the production of a novel biosurfactant promoting swarming motility in Pseudomonas aeruginosa: 3-(3-hydroxyalkanoyloxy)alkanoic acids (HAAs), the precursors of rhamnolipids. Microbiology 149, 2005-2013. doi: 10.1099/mic.0. 26154-0

Ditta, G., Stanfield, S., Corbin, D., and Helinski, D. R. (1980). Broad host range DNA cloning system for Gram-negative bacteria: construction of a gene bank of Rhizobium meliloti. Proc. Natl. Acad. Sci. U.S.A. 77, 7347-7351. doi: 10.1073/ pnas.77.12.7347

Domínguez-Cuevas, P., González-Pastor, J. E., Marqués, S., Ramos, J. L., and De Lorenzo, V. (2006). Transcriptional tradeoff between metabolic and stressresponse programs in Pseudomonas putida KT2440 cells exposed to toluene. J. Biol. Chem. 281, 11981-11991. doi: 10.1074/jbc.M509848200

Dusane, D. H., Zinjarde, S. S., Venugopalan, V. P., McLean, R. J. C., Weber, M. M., and Rahman, P. K. S. M. (2010). Quorum sensing: implications on rhamnolipid biosurfactant production. Biotechnol. Genet. Eng. Rev. 27, 159-184. doi: 10. 1080/02648725.2010.10648149

Duwe, A., Tippkötter, N., and Ulber, R. (2019). Lignocellulose-biorefinery: ethanolfocused. Adv. Biochem. Eng. Biotechnol. 166, 177-215. doi: 10.1007/10_2016_ 72

Edwards, J. R., and Hayashi, J. A. (1965). Structure of a rhamnolipid from Pseudomonas aeruginosa. Arch. Biochem. Biophys. 111, 415-421. doi: 10.1016/ 0003-9861(65)90204-3

Gehring, C., Wessel, M., Schaffer, S., and Thum, O. (2016). The power of biocatalysis: a one-pot total synthesis of rhamnolipids from butane as the sole carbon and energy source. ChemistryOpen 5, 513-516. doi: 10.1002/open. 201600127

Germer, A., Tiso, T., Müller, C., Behrens, B., Vosse, C., Scholz, K., et al. (2020). Exploiting the natural diversity of RhlA acyltransferases for the synthesis of the rhamnolipid precursor 3-(3-hydroxyalkanoyloxy)alkanoic acid. Appl. Environ. Microbiol. 86, 1-16. doi: 10.1128/AEM.02317-19

Gibson, D. G., Young, L., Chuang, R. Y., Venter, J. C., Hutchison, C. A., and Smith, H. O. (2009). Enzymatic assembly of DNA molecules up to several hundred kilobases. Nat. Methods 6, 343-345. doi: 10.1038/nmeth.1318

Gliese, N., Khodaverdi, V., Schobert, M., and Görisch, H. (2004). AgmR controls transcription of a regulon with several operons essential for ethanol oxidation in Pseudomonas aeruginosa ATCC 17933. Microbiology 150, 1851-1857. doi: $10.1099 / \mathrm{mic} .0 .26882-0$

Görisch, H. (2003). The ethanol oxidation system and its regulation in Pseudomonas aeruginosa. Biochim. Biophys. Acta Proteins Proteomics 1647, 98-102. doi: 10.1016/S1570-9639(03)00066-9

Gudiña, E. J., Rodrigues, A. I., de Freitas, V., Azevedo, Z., Teixeira, J. A., and Rodrigues, L. R. (2016). Valorization of agro-industrial wastes towards the 
production of rhamnolipids. Bioresour. Technol. 212, 144-150. doi: 10.1016/j. biortech.2016.04.027

Hanahan, D. (1983). Studies on transformation of Escherichia coli with plasmids. J. Mol. Biol. 166, 557-580. doi: 10.1016/S0022-2836(83)80284-8

Heipieper, H. J., Weber, F. J., Sikkema, J., Keweloh, H., and de Bont, J. A. M. (1994). Mechanisms of resistance of whole cells to toxic organic solvents. Trends Biotechnol. 12, 409-415. doi: 10.1016/0167-7799(94)90029-9

Hempel, N., Görisch, H., and Mern, D. S. (2013). Gene $\operatorname{ercA}$, encoding a putative iron-containing alcohol dehydrogenase, is involved in regulation of ethanol utilization in Pseudomonas aeruginosa. J. Bacteriol. 195, 3925-3932. doi: 10. 1128/JB.00531-13

Henkel, M., Müller, M. M., Kügler, J. H., Lovaglio, R. B., Contiero, J., Syldatk, C., et al. (2012). Rhamnolipids as biosurfactants from renewable resources: concepts for next-generation rhamnolipid production. Process Biochem. 47, 1207-1219. doi: 10.1016/j.procbio.2012.04.018

Jarvis, F. G., and Johnson, M. J. (1949). A glyco-lipide produced by Pseudomonas aeruginosa. J. Am. Chem. Soc. 71, 4124-4126. doi: 10.1021/ja01180a073

Johann, S., Seiler, T. B., Tiso, T., Bluhm, K., Blank, L. M., and Hollert, H. (2016). Mechanism-specific and whole-organism ecotoxicity of mono-rhamnolipids. Sci. Total Environ. 548-549, 155-163. doi: 10.1016/j.scitotenv.2016.01.066

Junker, B. (2007). Foam and its mitigation in fermentation systems. Biotechnol. Prog. 23, 767-784. doi: 10.1021/bp070032r

Kuhn, D., Blank, L. M., Schmid, A., and Bühler, B. (2010). Systems biotechnology rational whole-cell biocatalyst and bioprocess design. Eng. Life Sci. 10, 384-397. doi: 10.1002/elsc.201000009

Lang, S., and Wullbrandt, D. (1999). Rhamnose lipids - biosynthesis, microbial production and application potential. Appl. Microbiol. Biotechnol. 51, 22-32. doi: $10.1007 / \mathrm{s} 002530051358$

LanzaTech (2018). World's First Commercial Waste Gas to Ethanol Plant Starts up. Available online at: https://www.lanzatech.com/2018/06/08/worlds-firstcommercial-waste-gas-ethanol-plant-starts/ (accessed May 11, 2020).

Li, W., Jayakody, L. N., Franden, M. A., Wehrmann, M., Daun, T., Hauer, B., et al. (2019). Laboratory evolution reveals the metabolic and regulatory basis of ethylene glycol metabolism by Pseudomonas putida KT2440. Environ. Microbiol. 21, 3669-3682. doi: 10.1111/1462-2920.14703

López-Sánchez, A., Leal-Morales, A., Jiménez-Díaz, L., Platero, A. I., BardalloPérez, J., Díaz-Romero, A., et al. (2016). Biofilm formation-defective mutants in Pseudomonas putida. FEMS Microbiol. Lett. 363:fnw127. doi: 10.1093/femsle/ fnw127

Maier, R. M., and Soberón-Chávez, G. (2000). Pseudomonas aeruginosa rhamnolipids: biosynthesis and potential applications. Appl. Microbiol. Biotechnol. 54, 625-633. doi: 10.1007/s002530000443

Martínez-García, E., and de Lorenzo, V. (2011). Engineering multiple genomic deletions in Gram-negative bacteria: analysis of the multi-resistant antibiotic profile of Pseudomonas putida KT2440. Environ. Microbiol. 13, 2702-2716. doi: $10.1111 / j .1462-2920.2011 .02538 . x$

Matsufuji, M., Nakata, K., and Yoshimoto, A. (1997). High production of rhamnolipids by Pseudomonas aeruginosa growing on ethanol. Biotechnol. Lett. 19, 1213-1215. doi: 10.1023/A:1018489905076

McKenna, A., Hanna, M., Banks, E., Sivachenko, A., Cibulskis, K., Kernytsky, A., et al. (2010). The genome analysis toolkit: a MapReduce framework for analyzing next-generation DNA sequencing data. Genome Res. 20, 1297-1303. doi: $10.1101 /$ gr.107524.110

Molina-Santiago, C., Udaondo, Z., Gómez-Lozano, M., Molin, S., and Ramos, J. L. (2017). Global transcriptional response of solvent-sensitive and solventtolerant Pseudomonas putida strains exposed to toluene. Environ. Microbiol. 19, 645-658. doi: 10.1111/1462-2920.13585

Müller, M. M., Hörmann, B., Kugel, M., Syldatk, C., and Hausmann, R. (2011). Evaluation of rhamnolipid production capacity of Pseudomonas aeruginosa PAO1 in comparison to the rhamnolipid over-producer strains DSM 7108 and DSM 2874. Appl. Microbiol. Biotechnol. 89, 585-592. doi: 10.1007/s00253-0102901-z

Navarrete, B., Leal-Morales, A., Serrano-Ron, L., Sarrió, M., Jiménez-Fernández, A., Jiménez-Díaz, L., et al. (2019). Transcriptional organization, regulation and functional analysis of $f h F$ and fleN in Pseudomonas putida. PLoS One 14:e0214166. doi: 10.1371/journal.pone.0214166

Nelson, K. E., Weinel, C., Paulsen, I. T., Dodson, R. J., Hilbert, H., Martins dos Santos, V. A. P., et al. (2002). Complete genome sequence and comparative analysis of the metabolically versatile Pseudomonas putida KT2440. Environ. Microbiol. 4, 799-808. doi: 10.1046/j.1462-2920.2002.00366.x

Nitschke, M., Costa, S. G. V. A. O., and Contiero, J. (2011). Rhamnolipids and PHAs: recent reports on Pseudomonas-derived molecules of increasing industrial interest. Process Biochem. 46, 621-630. doi: 10.1016/j.procbio.2010. 12.012

Nogales, J., Mueller, J., Gudmundsson, S., Canalejo, F. J., Duque, E., Monk, J., et al. (2020). High-quality genome-scale metabolic modelling of Pseudomonas putida highlights its broad metabolic capabilities. Environ. Microbiol. 22, 255-269. doi: $10.1111 / 1462-2920.14843$

Ochsner, U. A., Fiechter, A., and Reiser, J. (1994). Isolation, characterization, and expression in Escherichia coli of the Pseudomonas aeruginosa rhlAB genes encoding a rhamnosyltransferase involved in rhamnolipid biosurfactant synthesis. J. Biol. Chem. 269, 19787-19795.

Ochsner, U. A., Reiser, J., Fiechter, A., and Witholt, B. (1995). Production of Pseudomonas aeruginosa rhamnolipid biosurfactants in heterologous hosts. Appl. Environ. Microbiol. 61, 3503-3506. doi: 10.1128/aem.61.9.3503-3506. 1995

Park, J. O., Liu, N., Holinski, K. M., Emerson, D. F., Qiao, K., Woolston, B. M., et al. (2019). Synergistic substrate cofeeding stimulates reductive metabolism. Nat. Metab. 1, 643-651. doi: 10.1038/s42255-019-0077-0

Rahim, R., Burrows, L. L., Monteiro, M. A., Perry, M. B., and Lam, J. S. (2000). Involvement of the $r m l$ locus in core oligosaccharide and O polysaccharide assembly in Pseudomonas aeruginosa. Microbiology 146, 2803-2814. doi: 10. 1099/00221287-146-11-2803

Rahim, R., Ochsner, U. A., Olvera, C., Graninger, M., Messner, P., Lam, J. S., et al. (2001). Cloning and functional characterization of the Pseudomonas aeruginosa rhlC gene that encodes rhamnosyltransferase 2, an enzyme responsible for dirhamnolipid biosynthesis. Mol. Microbiol. 40, 708-718. doi: 10.1046/j.13652958.2001.02420.x

Ramos, J. L., Duque, E., Huertas, M. J., and Haïdour, A. (1995). Isolation and expansion of the catabolic potential of a Pseudomonas putida strain able to grow in the presence of high concentrations of aromatic hydrocarbons. J. Bacteriol. 177, 3911-3916. doi: 10.1128/jb.177.14.3911-3916. 1995

Robinson, J. T., Thorvaldsdóttir, H., Winckler, W., Guttman, M., Lander, E. S. Getz, G., et al. (2011). Integrative genomics viewer. Nat. Biotechnol. 29, 24-26. doi: 10.1038/nbt.1754

Rosales-Calderon, O., and Arantes, V. (2019). A review on commercial-scale highvalue products that can be produced alongside cellulosic ethanol. Biotechnol. Biofuels 12, 1-58. doi: 10.1186/s13068-019-1529-1

Sambrook, J., and Russell, D. W. (2001). Molecular cloning: A Laboratory Manual, 3rd Edn. New York, NY: Cold Spring Harbor Laboratory Press.

Santos, A. S., Sampaio, A. P. W., Vasquez, G. S., Santa Anna, L. M., Pereira, N., and Freire, D. M. G. (2002). Evaluation of different carbon and nitrogen sources in production of rhamnolipids by a strain of Pseudomonas aeruginosa. Appl. Biochem. Biotechnol. Part A Enzym. Eng. Biotechnol 98, 1025-1035. doi: 10.1385/ABAB:98-100:1-9:1025

Sha, R., Meng, Q., and Jiang, L. (2012). The addition of ethanol as defoamer in fermentation of rhamnolipids. J. Chem. Technol. Biotechnol. 87, 368-373. doi: $10.1002 / j \mathrm{ctb} .2728$

Silva, R., de, C. F. S., Almeida, D. G., Rufino, R. D., Luna, J. M., Santos, V. A., et al. (2014). Applications of biosurfactants in the petroleum industry and the remediation of oil spills. Int. J. Mol. Sci. 15, 12523-12542. doi: 10.3390/ ijms 150712523

Sim, L., Ward, O. P., and Li, Z. Y. (1997). Production and characterisation of a biosurfactant isolated from Pseudomonas aeruginosa UW-1. J. Ind. Microbiol. Biotechnol. 19, 232-238. doi: 10.1038/sj.jim.2900450

Soberón-Chávez, G., Lépine, F., and Déziel, E. (2005). Production of rhamnolipids by Pseudomonas aeruginosa. Appl. Microbiol. Biotechnol. 68, 718-725. doi: 10. 1007/s00253-005-0150-3

Solovyev, V., and Salamov, A. (2011). "Automatic annotation of microbial genomes and metagenomic sequences," in Metagenomics and its Applications in Agriculture, Biomedicine and Environmental Studies, ed. R. W. Li (Hauppauge, NY: Nova Science Publishers), 61-78.

Sudarsan, S., Dethlefsen, S., Blank, L. M., Siemann-Herzberg, M., and Schmid, A. (2014). The functional structure of central carbon metabolism in Pseudomonas 
putida KT2440. Appl. Environ. Microbiol. 80, 5292-5303. doi: 10.1128/AEM. 01643-14

Syldatk, C., Lang, S., Wray, V., and Witte, L. (1985). Chemical and physical characterization of four interfacial-active rhamnolipids from Pseudomonas spec. DSM 2874 grown on $\eta$-alkanes. Z. Naturforsch. C J. Biosci. 40, 51-60. doi: 10.1515/znc-1985-1-212

Tiso, T., Ihling, N., Kubicki, S., Biselli, A., Schonhoff, A., Bator, I., et al. (2020). Integration of genetic and process engineering for optimized rhamnolipid production using Pseudomonas putida. Front. Bioeng. Biotechnol. doi: 10.3389/ fbioe.2020.00976

Tiso, T., Wierckx, N., and Blank, L. M. (2014). "Non-pathogenic Pseudomonas as platform for industrial biocatalysis," in Industrial Bocatalysis, ed. P. Grunwald (Hoboken, NJ: Pan Stanford), 323-372.

Tiso, T., Zauter, R., Tulke, H., Leuchtle, B., Li, W.-J., Behrens, B., et al. (2017). Designer rhamnolipids by reduction of congener diversity: production and characterization. Microb. Cell Fact. 16:225. doi: 10.1186/s12934-017-0838-y

Vardar-Sukan, F. (1998). Foaming: consequences, prevention and destruction. Biotechnol. Adv. 16, 913-948. doi: 10.1016/S0734-9750(98)00010-X

Wang, Q., Fang, X., Bai, B., Liang, X., Shuler, P. J., Goddard, W. A., et al. (2007). Engineering bacteria for production of rhamnolipid as an agent for enhanced oil recovery. Biotechnol. Bioeng. 98, 842-853. doi: 10.1002/bit.21462

Wendisch, V. F., Brito, L. F., Gil Lopez, M., Hennig, G., Pfeifenschneider, J., Sgobba, E., et al. (2016). The flexible feedstock concept in industrial biotechnology: metabolic engineering of Escherichia coli, Corynebacterium glutamicum, Pseudomonas, Bacillus and yeast strains for access to alternative carbon sources. J. Biotechnol. 234, 139-157. doi: 10.1016/j.jbiotec.2016.07.022

Winterburn, J. B., Russell, A. B., and Martin, P. J. (2011). Integrated recirculating foam fractionation for the continuous recovery of biosurfactant from fermenters. Biochem. Eng. J. 54, 132-139. doi: 10.1016/j.bej.2011.02.011

Wittgens, A., Tiso, T., Arndt, T. T., Wenk, P., Hemmerich, J., Müller, C., et al. (2011). Growth independent rhamnolipid production from glucose using the non-pathogenic Pseudomonas putida KT2440. Microb. Cell Fact. 10, 1-17. doi: 10.1186/1475-2859-10-80

Wynands, B., Lenzen, C., Otto, M., Koch, F., Blank, L. M., and Wierckx, N. (2018). Metabolic engineering of Pseudomonas taiwanensis VLB120 with minimal genomic modifications for high-yield phenol production. Metab. Eng. 47, 121-133. doi: 10.1016/j.ymben.2018.03.011

Yang, J., Son, J. H., Kim, H., Cho, S., Na, J. G., Yeon, Y. J., et al. (2019). Mevalonate production from ethanol by direct conversion through acetyl-CoA using recombinant Pseudomonas putida, a novel biocatalyst for terpenoid production. Microb. Cell Fact. 18, 1-12. doi: 10.1186/s12934-0191213-y

Yousef-Coronado, F., Travieso, M. L., and Espinosa-Urgel, M. (2008). Different, overlapping mechanisms for colonization of abiotic and plant surfaces by Pseudomonas putida. FEMS Microbiol. Lett. 288, 118-124. doi: 10.1111/j.15746968.2008.01339.x

Zobel, S., Benedetti, I., Eisenbach, L., De Lorenzo, V., Wierckx, N., and Blank, L. M. (2015). Tn7-based device for calibrated heterologous gene expression in Pseudomonas putida. ACS Synth. Biol. 4, 1341-1351. doi: 10.1021/acssynbio. $5 \mathrm{~b} 00058$

Conflict of Interest: LB and TT declare that they are inventors of three related patents. (1) LB, F. Rosenau, S. Wilhelm, A. Wittgens, TT, "Means and methods for rhamnolipid production" HHU Düsseldorf University, TU Dortmund University, 2013 (WO 2013/041670 A1), (2) LB, B. Küpper, E. M. del Amor Villa, R. Wichmann, C. Nowacki, "Foam adsorption" TU Dortmund University, 2013 (WO 2013/087674 A1), and (3) LB, TT, A. Germer, "Extracellular production of designer hydroxyalkanoyloxy alkanoic acids with recombinant bacteria" RWTH Aachen University, 2015 (WO2017006252A1).

The remaining authors declare that the research was conducted in the absence of any commercial or financial relationships that could be construed as a potential conflict of interest.

Copyright (c) 2020 Bator, Karmainski, Tiso and Blank. This is an open-access article distributed under the terms of the Creative Commons Attribution License (CC BY). The use, distribution or reproduction in other forums is permitted, provided the original author(s) and the copyright owner(s) are credited and that the original publication in this journal is cited, in accordance with accepted academic practice. No use, distribution or reproduction is permitted which does not comply with these terms. 